\title{
DIE INSTITUTIONALISIERUNG DER ALEVITEN IN DEUTSCHLAND
}

\author{
Almanya'da Alevilerin Kurumsallaşma Sürecleri \\ Institutionalisation Processes of Alevis in Germany \\ Bülent KELEŞ *
}

Während mittlerweile 60 Jahre seit dem Beginn der Arbeitsmigration aus der Türkei nach Deutschland vergangen sind, gibt es in der Türkei leider nur eine überschaubare Anzahl an Publikationen über das Schicksal der Aleviten und ihren Existenzkampf in Deutschland, obwohl sie von vielen Forschern als die am stärksten von der Migration betroffene Gruppe angesehen werden. Dabei ließ sich der vielerseits als "alevitisches Erwachen" bezeichnete Existenzkampf der Aleviten im städtischen Kontext, der in den 1990er Jahren Fahrt aufnahm, erstmals in Deutschland beobachten. Im Rahmen dieser Studie wurde der Prozess der alevitischen Institutionalisierung in Deutschland untersucht, im Zuge dessen 1987 die erste offizielle alevitische Einrichtung entstand. In der Disziplin islamischen Konfessionsgeschichte zu verorten, legt dieser Artikel einen besonderen Fokus auf das Verhältnis von "Idee" und "Ereignis". Darüber hinaus wurden auch qualitative Forschungsmethoden aus dem Bereich der Sozialwissenschaften angewendet. Die Studie basiert auf teilnehmender Beobachtung und Interviews, die einen Zugang zu den Erfahrungen der befragten Personen gewähren sollten. In dieser Hinsicht wurde ein phänomenologischer Ansatz verfolgt. Auf Grundlage eines halbstrukturierten Fragebogens wurden Tiefeninterviews mit einem Großteil der Personen, die die alevitische Institutionalisierung in Deutschland eingeleitet haben, geführt, um

* Dr., Lehrer, Grundschule Goldbach, bulentkeles@hotmail.de, ORCID: 0000-0002-5397-7478 
den Institutionalisierungsprozess anhand der von ihnen gegebenen Informationen zu dokumentieren. Als Grundgesamtheit der Studie wurden die institutionellen Strukturen bestimmt, die mit dem Ziel entstanden sind, die in verschiedenen Städten Deutschlands lebenden Aleviten im religiösen Bereich zu repräsentieren, wobei die Personen, die an der Gründung dieser institutionellen Strukturen beteiligt waren oder derzeit in deren Führung sitzen, bewusst ausgewählt wurden. Bei der Durchführung des phänomenologischen Ansatzes wurde sehr stark darauf geachtet, das Vertrauen der Personen zu sichern, die eine wichtige Rolle bei der alevitischen Institutionalisierung gespielt haben (bzw. spielen) und das Phänomen der Studie bilden. Um ein vertrauensvolles Umfeld zu schaffen, stellte auch der Autor der Studie seine alevitische Identität in den Vordergrund. Die von den Interviewpartnern bereitgestellten Informationen und Dokumente über den institutionellen Prozess wurden mit den Informationen anderer Interviewpartner und den Angaben in Memoiren verglichen, um die Richtigkeit von Informationen zu prüfen.

Schlüsselwörter: Alevitische Institutionalisierung, Deutschland, Aleviten, Alevitentum, Dede-zentriert, Kulturzentriert. 


\section{ÖZ}

Türkiye'den Almanya'ya yönelik gerçekleşen işgücü göçünün üzerinden 60 yll geçmesine rağmen, birçok araştırmacının göçe en fazla maruz kalan grup olarak kabul ettiği Alevi vatandaşlarımıza ve onların Almanya'daki varoluş çabalarına yönelik Türkiye'de ne yazık ki çok fazla yayın bulunmamaktadır. Oysa 1990'lı yıllarla birlikte tırmanışa geçen ve birçok araştırmacının “alevi uyanışı” olarak isimlendirdiği Alevilerin kentsel ortamda varoluş mücadelelerinin ilk ortaya çıktığı ülke olarak Almanya ön plana çıkmaktadır. Bu çalışmada 1987'de ilk resmi kurumsal kimliğini ortaya koyan Almanya Alevi örgütlenme süreci incelenmiştir. Makale içerisinde İslam Mezhepleri Tarihi disiplini esas alınarak "fikir-hadise irtibatı” üzerinde özellikle durulmuştur. Bununla birlikte sosyal bilimler alanında kullanılan araştırma metotlarından da istifade edilmiştir. Bu çerçevede Almanya'daki Alevilerin kurumsallaşma sürecine yönelik gerçekleştirmiş olduğumuz çalışmada özellikle Nitel araştırma yöntemi kullanılmıştır. Katılımcı gözlem ve görüşme yöntemini benimseyip görüştügüumüz isimlerin deneyimlerinden yararlanma yolunu seçtiğimiz için de çalışmamız kapsamında nitel araştırma yönteminin fenomenolojik yaklaşımı tercih edilmiştir. Nitekim bu çerçevede Almanya'da ortaya çıkan Alevi kurumsallaşmasını başlatan isimlerin önemli bir bölümüyle yarı yapılandırılmış soru formu kullanılarak derinlemesine görüşmeler gerçekleştirilmiş ve örgütlenme süreci, onların verdikleri bilgiler üzerinden belgelenmeye çalışılmıştır. Araştırmamıza Evren olarak Almanya'nın değişik şehirlerinde yaşayan Alevileri dini alanda temsil maksadıyla ortaya çıkan kurumsal yapılar seçilmiş ve bu kurumsal yapıların kuruluş sürecinde görev almış veya halihazırda yönetiminde yer alan isimler, "amaçlı örneklem” olarak tercih edilmiştir. Fenomenolojik yaklaşım yürütülürken, fenomen olarak seçtiğimiz ve Alevi kurumsallaşmasında önemli roller üstlenmiş isimlerin tarafımıza güven duymasına azami derecede dikkat edilmeye çalışılmıştır. Güven ortamını sağlamak içinse Alevi kimliğimiz özellikle ön planda tutulmuştur. Görüşmecilerimizce tarafimıza iletilen kurumsal sürece dair bilgi ve belgeler, diğer görüşmecilerin vermiş oldukları bilgilerin yanı sıra hatırat kitaplarındaki anlatılanlarla da karşılaştırılarak doğru bilgiye ulaşılmaya çalışılmıştır.

Anahtar Kelimeler: Alevi Kurumsallaşması, Almanya, Aleviler, Alevilik, Dede Merkezci, Kültür Merkezci. 


\begin{abstract}
60 years after the labour migration from Turkey to Germany, unfortunately there are not many publications in Turkey towards Alevi citizens, whom many researchers consider to be the most exposed to migration, and their efforts to exist in Germany. However, Germany stands out as the country where the struggles of Alevis to exist in the urban environment, which many researchers call "the Alevi awakening" and which started to rise with the 1990's, first appeared. This paper analyses the German Alevi organisation process that revealed its first official corporate identity in 1987. The paper emphasizes the "idea-event connection" on the bases of the History of Islam's Denominations. It also uses research methods from social sciences. In this context, we especially used qualitative research method in our study on the institutionalisation process of Alevis in Germany. Since we adopted the participatory observation and interview method and benefited from the experiences of the persons we interviewed, we chose the phenomenological approach of qualitive research method within the scope of our study. Thus, in this context, we conducted in-depth interviews with a significant number of the names, who initiated the Alevi institutionalisation in Germany, using semi-structured questionnaire, and we tried to document the organisation process through the information they provided. For the study, we selected corporate structures emerged for the purpose of representing in the religious sphere Alevis living in different cities in Germany, and we chose as "purposive sample" the names who have taken part in the establishment process of these institutional structures or are already in their management. While carrying out the phenomenological approach, we tried to pay maximum attention to the trust of the names that we chose as phenomenon and who played important roles in the institutionalisation of Alevis. In order to provide a trust environment, we particularly prioritized our Alevi identity. We tried to reach the correct information by comparing information and documents about the institutional process obtained from our interviewers with the stories from the memoirs as well as the information given by other interviewers.
\end{abstract}

Keywords: Alevi institutionalisation, Germany, Alevis, Alevism, dede-centred, culture-centred. 


\section{Einleitung}

In seiner Untersuchung türkischer Organisationen in Deutschland stellt Erkan Perşembe die These auf, dass "kultureller Nationalismus" (2005: 127) bei der Institutionalisierung von Türken in Deutschland im Mittelpunkt stehe. Onur Bilge Kula unterteilt die türkischen Organisationen weiter entlang der Begriffe traditionalistisch und innovativ (2012: 173) und betont dabei, dass sich bei Türken in Deutschland ein Reflex zur Bewahrung der eigenen Identität beobachten ließe. Kulas Unterscheidung zufolge verabsolutiere der traditionalistische Flügel sein in der Türkei erworbenes Kulturverständnis und organisiere sich um dieses herum, während die Institutionalisierungsformen des innovativen Flügels auf einer Synthese der zukunftsorientierten und innovativen Elemente der traditionellen Kultur mit den Erfordernissen einer multikulturellen Gesellschaft beruhten. Kula wirft dem traditionalistischen Flügel vor, sich mehrheitlich der Religion zuzuwenden und zu radikalisieren, und lobt dementgegen den innovativen Flügel dafür, dass er säkulare Strukturen geschaffen habe. Doch unabhängig davon, ob sie mit dem Ziel der Bewahrung der Tradition oder auf der Suche nach Innovation entstanden seien, habe die Wahrung der eigenen Identität seit jeher den Hauptzweck dieser Organisationen dargestellt.

Der Begriff der Identität (Türkdoğan, 2007: 113; Akgün, 2018: 4) beschreibt, wie Gesellschaften, Bevölkerungsgruppen und Individuen sich selbst sehen und wie sie sich in historischen und sozialen Prozessen positionieren. In dieser Hinsicht steht der Begriff der Identität im direkten Zusammenhang mit den Fragen "Wer bist du?" und "Zu wem gehörst du?". Während die erste Frage über die persönliche Identität, die den Unterschied einer Person zu anderen offenbart, zu beantworten wäre, betrifft die zweite Frage die Identität der sozialen Gruppe, der diese Person angehört. So betrachtet beseitigt Identität Gefühle der Fremdheit, während sie aufgrund ihrer engen Beziehung zur Kultur den Menschen gleichzeitig eine Referenz bietet, um Phänomene, die sie wahrnehmen, zu begreifen und verstehen (Yap1c1, 2016: 4149). Unterdessen führt die Religion, angesichts ihrer unmittelbaren Bedeutung für die Frage nach der Identität des Menschen und danach, wer oder was der Mensch wirklich ist, zu einer Sakralisierung der Identität (Greil und Davidman, 2016: 3, 14). Auf individueller Ebene finden solche für heilig erklärten Identitäten in unterschiedlicher Weise Ausdruck, z.B. "Ich bin Muslim, ich bin Sunnit", "Ich bin Muslim und Hanafi", 
"Ich bin Türke und Muslim", "Ich bin Muslim und Alevit", "Ich bin Kurde und Alevit", "Ich bin Türke und Alevit" oder "Ich bin Alevit" usw.

Die Institutionalisierung von Aleviten vollzog sich grundsätzlich im Rahmen identitätszentrierter Organisationsformen. Bis zum Einsetzen einer Abwanderung von den Dörfern in die Städte in den 1950er/60er Jahren wiesen die Aleviten fast alle Merkmale der von Tönnies konzipierten Gemeinschaft (Tönnies, 1887: 9-45) auf. Im Kontext von Urbanisierung und Modernisierung kam es jedoch an den neuen Heimartorten zu einem Prozess der Auflösung. Dieser war hauptsächlich darauf zurückzuführen, dass sich die Dede-Talip-Beziehung in den modernen urbanen Verhältnissen, in denen die Lebenswelten von Sunniten und Aleviten eng miteinander verwoben waren, nicht aufrechterhalten ließ (Şahin, 2002: 130). Dieser Prozess impliziert also einen Übergang von einer homogenen Gemeinschaft zur Gesellschaft, in der die Grenzen zwischen verschiedenen Lebensformen deutlicher zutage treten. Der Übergang von der Gemeinschaft zur Gesellschaft bedeutet nicht nur Fortschritt und Urbanisierung, sondern sorgt zudem für einen Verlust der der Tradition eigenen solidarischen und aufrichtigen Bindungen (Türkdoğan, 2007: 54). Mit Blick auf die Aleviten führte diese Entwicklung zu größerer Sichtbarkeit und Partizipation in verschiedenen gesellschaftlichen Bereichen, von der Politik über die Kunst bis hin zur Wissenschaft und Wirtschaft. Aleviten in Deutschland und der Türkei waren dabei darauf bedacht, einer durch den Einfluss verschiedener Religionen und Kulturen bedingten Assimilation zu entgehen, und schlossen sich daher unter dem Dach von Vereinigungen zusammen, die unter der Leitung besonders qualifizierter alevitischer Persönlichkeiten gegründet wurden. Diese Zusammenkünfte, die bis in die 1980er Jahre einen politisch heterogenen Charakter aufwiesen und an denen auch die (türkische) Einheitspartei beteiligt war, institutionalisierten sich im Zuge der Gründung des ersten Vereins 1987 in Köln zu einer religiös orientierten Organisation, die allerdings nicht als rein religiöse Gruppe $e^{2}$ bestehen blieb. Vor allem durch die Beteiligung

1 Während Tönnies den Begriff der Gesellschaft verwendet, um urbanisierte Kollektive zu beschreiben, deren interne Beziehungen nach meritokratischen Prinzipien geregelt sind, definiert er Gemeinschaft als Kollektive, in denen Erbhierarchien im Vordergrund stehen. Siehe Tönnies, 1887: 46-95.

2 Rein religiöse Gruppen bezeichnen Kollektive, deren Mitglieder durch religiöse Zugehörigkeit miteinander verbunden sind. Für ausführliche Informationen siehe Günay, 2012: 276; Akyüz, 2007: 54-58. 
von Personen aus dem linken Spektrum gewannen ab 1990 kulturell orientierte Formen der Institutionalisierung unter den Aleviten an Gewicht.

Bei der Institutionalisierung alevitischen Lebens nach 1980 handelt es sich um eine zweigliedrige Struktur, die mehrere Vereine umfasst. Den Aleviten, die sich um die Dedes herum organisierten, standen andererseits politisch erfahrenere und organisierte, säkular eingestellte Aleviten gegenüber, die sich infolge eines Paradigmenwechsels in der Linken zu einer kulturellen Verständnis von Identität hin orientierten. ${ }^{3}$ Der zumeist von den Dedes mitbegründete Typ von Organisationen war dabei geprägt von der Sehnsucht nach dem ursprünglich in den Dörfern gelebten traditionellen Alevitentum und verfolgte mittels Institutionalisierung das Ziel, ebenjenes traditionelle Alevitentum wiederherzustellen (Kehl-Bodrogi, 1996: 52) und am Leben zu erhalten. Dieser Organisationstypus soll hier als Dedezentriert bezeichnet werden. Der zweite Typ von Organisationen, der auf einem von Massicard als "Alevitismus"4 beschriebenen Verständnis basiert, kann dagegen passenderweise als kulturzentriert gefasst werden. Bei diesen Organisationen, die von Personen aus dem linken Spektrum angeführt wurden und einen Identitätswandel (Kehl-Bodrogi, 1996: 52) einläuteten, standen die Förderung des Alevitentums durch kulturelle Aktivitäten und die soziokulturelle Funktion der geschaffenen Institution im Vordergrund. In diesem Rahmen wurden angesichts lauter werdender Forderungen mit der Zeit diverse Vereine gegründet. Wurde im ersten Fall das Alevitentum selbst als Zweck betrachtet, sah man es im zweiten als Mittel. So will der Dede-zentrierten Flügel das Bewusstsein für das Alevitentum wiederbeleben und letztere als heilige Identität ausleben, während der kulturzentrierte Flügel von "linken" und "demokratischen" Personen angeführt wird, die die Aleviten politisch instrumentalisieren wollen (Rittersberger-T1lıç, 2010: 100). Ein weiterer Grund für

3 In dieser Hinsicht ähnelt dieser Unterschied jenem zwischen der Republikanischen Bildungsstiftung (Cem-Stiftung) und dem Pir Sultan Abdal Kulturverein (PSAKD) in der Türkei. Während die Dede-zentrierten Organisationen an die Cem-Stiftung erinnern, stehen die kulturzentrierten Organisationen dem PSAKD näher. Zur Cem-Stiftung und dem PSDAK siehe Okan, 2004: 125-189; Ertan, 2017: 174-229. Massicard unterscheidet zudem zwischen religiösen und säkularen Aleviten. Siehe Massicard, 2007: 169.

4 Massicard bezeichnet den Alevitismus als "eine Bewegung vorwiegend politischer Natur, die im Namen des Alevitentum mobilisiert und dieses zu einer Mission erhebt und rationalisiert. Der Ursprung dieser Bewegung, die von modernen Organisationen, hauptsächlich Vereinen, getragen wird, liegt in 1960er Jahren, doch erst ab Ende der 1980er Jahre wurde sie wirklich wahrgenommen." Siehe Massicard, 2007: 21. 
die Unterteilung der Organisationsstrukturen in diesem Artikel ist die Tatsache, dass die Mehrheit derjenigen, die sich um die kulturzentrierten Strukturen versammeln, in der Vergangenheit sowohl in der türkischen Einheitspartei als auch in marxistischen/ linken Gruppierungen politisch aktiv waren. Mit Blick auf in der Türkei durchgeführte Studien kann gesagt werden, dass die kulturzentrierten Strukturen in der Nähe von Rıza Yıldırıms Definition des modernen Alevitentums zu verorten sind, während sich in den Dede-zentrierten Institutionen die Nostalgie des traditionellen Alevitentums widerspiegelt (2012: 143). In Dede-zentrierten Institutionen sind die Dedes in der Autoritätshierarchie über dem Vorstand angesiedelt, selbst wenn sie selbst keine führende Position besetzen. ${ }^{5}$ Mit dem Verweis auf die diversen Vereine, die die Selbstorganisation von Aleviten in Deutschland umfasst, soll darauf hingewiesen werden, dass sich diese Organisationen, die sich in zwei unterschiedliche Richtungen entwickelt haben, verschiedenen, voneinander unabhängigen Dachverbänden angeschlossen sind, von denen als stärkster die Alevitische Gemeinde Deutschland zu nennen ist.

Akyüz stellt fest, dass gegenseitige Beziehungen und Kommunikation eine wichtige Rolle bei der Klassifizierung von Gruppen spielen (2007: 54-58). Perşembe bestätigt diese Feststellung im Zusammenhang mit türkischen Organisationen in Deutschland und führt aus, dass sich die Türken, die nach 1961 ihr Heimatland verließen, im Ankunftsland in verschiedenen soziokulturellen Gruppen zusammenzuschließen begannen. Er weist dabei auch auf die wichtige Rolle hin, die verschiedene Faktoren vom Bildungsniveau bis zur ideologischen Überzeugung und der Herkunft aus denselben Regionen in der Türkei in diesen Zusammenschlüssen spielten (2005: 14).Zweifellos hat sich der Institutionalisierungsprozess von Aleviten unter dem Einfluss ähnlicher Faktoren vollzogen, d.h. ideologische Überzeugungen, religiöse Zugehörigkeit und kulturelles Umfeld haben die Organisierungsstrategien von Aleviten beeinflusst. Unter Berücksichtigung all dieser Faktoren und der oben erwähnten zweigliedrigen Struktur soll Institutionalisierung von Aleviten in Deutschland im Folgenden in zwei separaten Abschnitten, nämlich Dede-zentrierte und kulturzentrierte Institutionalisierung, anhand diverser Vereine untersucht werden.

5 Cemal Salman, der diese Frage in Bezug auf das Kölner HBV Cemevi betrachtet, erklärt, dass es dort einen Dede-Rat gebe und dass dieser Rat aufgrund einer Art ungeschriebenen Gesetzes größere Befugnisse als der Vereinsvorstand und zudem Immunität besitze. Siehe Salman, 2015: 400. 


\section{Dede-zentrierte Institutionalisierung}

Obwohl den Vereinen, die sich in den 1970er Jahren in der Föderation der Patriotischen Vereinigung zusammenschlossen, unter anderem auch viele Aleviten angehörten, können diese nicht als alevitische institutionelle Strukturen betrachten werden, da bei ihnen politische Motive im Vordergrund standen. Demgegenüber soll hier die Meinung vertreten werden, dass die ersten Beispiele für die Institutionalisierung von Aleviten in Deutschland die hier als Dede-zentriert bezeichneten Organisationen darstellen, die in den 1980er Jahren auf Initiative von Şinasi Koç Dede aufkeimten und hier und dort bis heute fortexistieren. Da Religion und traditionelle Identitätsbilder für ihre Mitglieder und deren vereinsinternen Rollen von vorrangiger Bedeutung sind, ähneln die Dede-zentrierten Organisationen als Kollektiv der Form nach der Gemeinschaft (Akyüz, 2007: 21, 22) , an der das Individuum in all seinen Facetten partizipiert. Wenngleich in modernen, bzw. Modernisierungsprozesse durchlaufenden Gesellschaften unmöglich länger von Gemeinschaften gesprochen werden kann, kann zur Erklärung angeführt werden, dass die Nostalgie nach der Vergangenheit ausschlaggebend für eine Orientierung hin zu solchen traditionalistischen Strukturen ist. Orhan Türkdoğan beschreibt anhand des Bildes einer "Gemeinschaft in der Gesellschaft', wie der Multikulturalismus in modernen Städten zur Tribalisierung bestimmter Gesellschaftsgruppen geführt habe (2007: 27). Der vorliegende Artikel stützt sich bei der Klassifizierung der Dede-zentrierten Strukturen auf diese, im Allgemeinen für plausibel befundene Beobachtung Türkdoğans. Demzufolge werden die Dede-zentrierten Formen der Institutionalisierung in den Stadtzentren hier als Ergebnis der Bestrebungen von Personen betrachtet, die die Tradition, wenn auch nur partiell, noch selbst erlebt hatten, einen beiligen Ort zum Ausleben religiöser Praktiken zu schaffen. Zeynel Gül, der sich mit dieser Suche nach einem heiligen Ort beschäftigt, sieht in der in den ersten Vereinen angewandte Regel, dass die Schuhe vor dem Betreten der Räumlichkeiten ausgezogen werden mussten, eine Nachahmung von Moscheevereinen (1999: 89). Tatsächlich jedoch unternahmen diese Organisationen, die innerhalb der alevitischen Institutionen insbesondere durch ihre Sehnsucht nach dem traditionellem Alevitentum hervorstachen, größte Anstrengungen, um die tradierten Werte in dem modernen städtischen Kontext zu bewahren, und versuchten das, was sie aus ihren Dörfern kannten, in die neugeschaffenen heiligen Orte zu tragen und dort zu leben. Diesbezüglich kommt Kehl-Bodrogis Feststellung hinsichtlich

6 Zur Unterscheidung von Gemeinschaft und Gesellschaft siehe Tönnies, 1987: 9-95. 
einer "Wiederherstellung des Ansehens der Dedes" (1996: 52). eine zentrale Bedeutung zu. Diese Feststellung deutet in gewisser Weise auf eine Wiederentdeckung der in die Ferne geratenen religiösen und kulturellen Werte hin und unterstreicht dabei, wie wichtig es ist, die Dedes in ihrem Versuch, die Tradition aller Schwierigkeiten zum Trotz am Leben zu erhalten, zu unterstützen. Dass es Şinasi Koç war, der diesen Prozess eingeleitet hat, heißt nicht gleichzeitig, dass sämtlich Organisationen seiner Kontrolle unterstehen. So spalteten beispielsweise die Debatten um die Fragen "Wurde das Opfer für Ismael oder Isaak geschickt?" und "Fand das ,Kerbela-Ereignis" im April oder im Oktober statt?” (Şahhüseyinoğlu, 2001: 86, 88) Die Dede-zentrierten Organisationen in zwei Lager, von denen sich das eine um die Föderation scharte, während das andere der publizistischen Linie des Gerçek İlim Dergisi ("Zeitschrift für Wahre Wissenschaft") folgte. Im Zuge dieser Spaltung schlossen sich die meisten Vereine, die das traditionelle Alevitentum in den Mittelpunkt stellten, der Föderation der Alevitischen Gemeinden (FAG) an. Der Einflussbereich von Şinasi Koç dagegen beschränkte sich in der Folge auf diejenigen Vereine, die sich im Umfeld der Zeitschrift für Wabre Wissenschaft bewegten.

In dieser Phase lag das Hauptanliegen in einer Verabsolutierung (Kula, 2012: 173) der in der Türkei erworbenen traditionellen Kultur und der Wahrung des traditionellen Religionsverständnisses. Die Institutionalisierung der Dede-zentrierten Organisationen, die sich bislang in Privathäusern und/oder kurzfristig angemieteten Räumen zusammengefunden hatten, war hauptsächlich von dem Ziel geleitet, einen Ort, wenn möglich gar ein großes Gebäude zu haben, an dem der Glaube praktiziert werden konnte. Nach Erreichen dieses Hauptziels entwickelten sich diese Orte zu Einrichtungen, in denen sozialer Austausch und kultureller Transfer stattfanden. Für die Dede-zentrierten Organisationen ist das Alevitentum nicht unabhängig vom Islam. Ihre Mitglieder sind vielfach traditionsverbundene Aleviten, die Yildirim folgendermaßen beschreibt: "Wenn Kerbela erwähnt wird, ergreift sie tiefe Traurigkeit, wenn ,Murteza Ali' erwähnt wird, zittert ihr Herz und sie wehren sich entschieden gegen noch die kleinste Veränderung des Cem-Rituals" (Y1ldırım, 2012: 143). In Anbetracht der Tatsache, dass sie um die männlichen Angehörigen der Ocak-Familien (Ocakzade) herum organisiert sind, welche väterlicherseits von der Ehl-i Beyt abstammen, lassen sich die Dedezentrierten Vereine in der Tat innerhalb des Islams verorten. Ihre enge Verbindung mit dem Islam muss auch dem Journalisten Zeynel Gül aufgefallen sein. Zumindest ließe sich so seine etwas überspitzte Kritik erklären, dass “... sie einen Koran vor sich haben und versuchen, einen Vers in jede aktuelle Diskussion einqubringen... wobei jeder Vers, den 
einer der Dedes anführte, von einem anderen Dede abgelehnt wird, der wiederum behauptet, dass diese Bedeutung in einem anderen Vers zu finden sei." (2002: 373; 1999: 87) ${ }^{7}$ Wenn auch nicht in diesem Ausmaß, so konnte doch auch im Rahmen dieser Arbeit beobachtet werden, dass die Dede-zentrierten Organisationen primär darauf bedacht sind, ihren Glauben zu bewahren, und sich im Rahmen ihrer Institutionalisierung und bei der Ausrichtung religiöser Veranstaltungen am traditionellen Alevitentum orientieren und diese Tradition lebendig halten wollen. Dementsprechend nimmt der Koran einen wichtigen Platz unter den Quellen des traditionellen Alevitentums ein. Die Dede-zentrierten Organisationen sind äußerst sensibel, was den Islam und seine Heiligkeiten, allen voran den Koran, die Propheten und die Engel, betrifft. Szenen, wie sie Gül behauptet beobachtet zu haben, sind allerdings bislang nirgends anders dokumentiert worden. Eine Perspektive, die teils mit der von Gül deckt, lässt sich eventuell bei Ali Rıza Gülçiçek finden, einem der Mitbegründer der kulturzentrierten Organisation in Köln. Dieser erklärt, dass er die Dede-zentrierten Organisationen, die die Föderation Alevitischer Gemeinden gegründet hatten, für konservativ halte (2018: 69). Sofern Gülçiçek an dieser Stelle mit Konservatismus Religiosität meint, kann man ihm getrost Recht geben. Denn die in den Dede-zentrierten Institutionen organisiert Veranstaltungen sind überwiegend religiöser Natur. Wahrscheinlich ist es dieser Umstand, auf den Massicard aufmerksam machen will, indem sie hervorhebt, dass religiöse Aleviten in den von ihnen organisierten Veranstaltungen Dedes als Redner auftreten ließen (2007: 169).

Die hier als Hüter des traditionellen Alevitentums begriffenen Dede-zentrierten Organisationen müssen sich die in gewisser Hinsicht berechtigte Kritik gefallen lassen, den Ocak-zentrierten Ansatz des traditionellen Alevitentums nicht beizubehalten haben (bzw. es nicht geschafft zu haben, diesen beizubehalten). Tatsächlich trifft man nicht nur in Dede-zentrierten Organisationen, sondern in allen alevitischen Institutionen, die in den Stadtzentren entstanden sind, einschließlich der Cemevis in der Türkei, häufig auf Dedes, die Aleviten betreuen, die nicht ihre eigenen Talips sind, und auf Aleviten, die sich an Ocakzades binden, die nicht ihre eigenen Dedes sind. An dieser Stelle darf nicht außer Acht gelassen werden, dass es, wie auch İsmail Engin festgestellt hat, mit der Migration zu Brüchen in der Dede-Talip-Beziehung kam und die Ocaks ihre Funktion einzubüßen begannen. Engin führt aus, dass Talips, die das

7 Auch Sökefeld ist aufgefallen, dass die Dedes den Kuran als Quelle betrachten. Siehe Sökefeld, 2008a: 202, 203. 
Alevitentum trotz allem fortsetzen wollten, es vorzögen, sich anstelle des Dedes und Ocaks, dem sie angehörten, als das traditionelle Alevitentum noch Bestand hatte, dem im jeweiligen Verein oder Cemevi anwesenden Dede anzuschließen (2001: 84). So versuche man, die Tradition am Leben zu halten, in dem man sie abwandelt.

Interessanterweise argumentiert Kehl-Bodrogi, dass die Dede-zentrierten Organisationen als Alternative zu den nach dem Vorbild der Föderation der Patriotischen Vereinigungen (Yurtseverler Birliği Federasyonu, YBF) gegründeten Vereinen entstanden seien (2002: 39). Hält man sich jedoch vor Augen, dass es zu Zeiten der Gründung dieser nach Hacı Bektaş Veli benannten Vereine nur in Berlin und München aktive Vereine gab, die unter dem Namen Patriotische Vereinigung liefen, scheint die These, dass es sich bei den zunächst in Köln, Ahlen, Mainz und Augsburg entstehenden Hacı Bektaş Veli Kultur- und Fördervereinen um alternative oder rivalisierende Vereine zu Vereinen in kilometerweit entfernten Städten gehandelt haben soll, kaum plausibel. Gänzlich entkräftet wird diese Behauptung angesichts der Tatsache, dass sich die Münchner Patriotische Vereinigung, die bis 1990 aktiv blieb, in der Folge entlang des traditionellen Alevitentums reorganisierte und in das Münchner Türkisch-Alevitisch-Bektaschitisches Kulturzentrum umbenannte. Was Kehl Bodrogi auf diese Idee brachte, dürfte eventuell eine Spaltung in Berlin sein. Die dortige Patriotische Vereinigung benannte sich 1990 in das Anatolisch-Alevitische Kulturzentrum Cemevi Berlin um. Aufgrund von Meinungsverschiedenheiten in Bezug auf die korrekte Auffassung vom Alevitentum kam es hier 1997 zum Bruch, der zur Entstehung eines alternativen Vereins, nämlich dem Hacı Bektaş Veli Kulturverein, führte (Massicard, 2007: 304).

Der Umstand, dass die ersten Dede-zentrierten Vereine sich beispielsweise Hac1 Bektaş Veli Verein oder Bektaschi Verein nannten, obwohl doch, wie auch in ihren Satzungen explizit zum Ausdruck kam, Alevitentum und Aleviten im Mittelpunkt ihrer Arbeit standen, sollte nicht unabhängig vom Urbanisierungsprozess betrachtet werden. Gilt der Bektaschismus doch als städtisch/urban. Obwohl die Bektaschi-Orden durch einen Beschluss im Jahre 1826 verboten und ihre Anhänger anschließend verfolgt wurden ${ }^{8}$, sind beide noch heute als geistreich und respektabel konnotiert. Deshalb werden der Bektaschismus und die Bektaschiten in der Türkei als vergleichsweise weniger fremd betrachtet als das Alevitentum und die Aleviten, die als das 'andere' des sunnitischen Islam gelten (Sökefeld, 2015: 406;

8 Für eine umfangreiche Studie zu diesem Thema siehe Soyyer, 2005: 59-96. 
Üçer, 2010: 463). Ebenso scheint die Tatsache, dass Hacı Bektaş Veli als ein von allen Gesellschaftsgruppen in der Türkei akzeptierter, einheitsstiftender Heiliger gilt, die in Deutschland lebenden Aleviten dazu veranlasst zu haben, sich um seinen Namen herum zu organisieren. In den frühen Phasen der Urbanisierung zogen es die Kizilbasch/Aleviten vor, sich eher als Bektaschi denn als Alevi zu bezeichnen, wenn sie in den Stadtzentren ihre religiöse Identität bekundeten. Mittels der Namen, den sie ihren Vereinen gaben, wollten die Aleviten offensichtlich ihre religiöse Identität legitimieren. Es ist davon auszugehen, dass sie Marginalisierungserfahrungen aufgrund des Begriffes "Alevi” zuvorkommen wollten und deshalb Namen wie etwa Hacı Bektaş Veli oder Bektaschi wählten, da Hacı Bektaş Veli auch in der sunnitischen Tradition als Glaubensführer anerkannt wird.

Die Dede-zentrierten Organisationen zeichnen sich weiterhin dadurch aus, dass sie keine Distanz zum türkischen Staat und seinen Institutionen wahren. Grundsätzlich befürworten die Vereine das Aufhängen von Türkei-Fahnen und Atatürk-Postern in ihren Räumlichkeiten; mancherorts gelten diese gar als rote Linie. Im Rahmen von Diskussionen darüber, dass Atatürk-Porträts die Cems begleiteten und die Wände der Cemevis schmückten, wurde unter anderem die Position vertreten, dass Atatürk selbst Alevit gewesen sei. Es mag sein, dass dieser Glaube sich einer unschuldigen Äußerung verdankt, die sich auf die guten Beziehungen zwischen den Bektaschis und dem Komitee für Union und Fortschritt (Maden, 2015: 185-213), dem auch Atatürk angehörte, stützte. Allerdings sollte in der Sache auch der Beitrag berücksichtigt werden, den das Treffen zwischen Atatürk und Çelebi Cemalettin während Atatürks Besuchs in Hacıbektaş am 22. und 23. Dezember 1919 eventuell geleistet haben mag. ${ }^{9}$ Obwohl der eigentliche Anlass des Treffens Atatürks Absicht war, Unterstützung für den Unabhängigkeitskampf zu gewinnen, kursieren unter Aleviten deutlich weitreichendere Meinungen zu diesem Thema. Demzufolge habe es ein privates Treffen zwischen Atatürk und Salih Niyazi Baba und Çelebi Cemalettin gegeben, bei dem ein "Ayin-i Cem” abgehalten worden sei, in dem Atatürk ein "Versprechen" (ikerar) abgelegt und somit "den Weg beschritten habe" (yola kabul) habe, d.h., aufgenommen worden sei (Şener, 1994: 63-68; Öz, 1990: 46-49). ${ }^{10}$

9 Für eine wissenschaftliche Arbeit bezüglich dieses Besuchs siehe Küçük, 2003: 128-130.

10 Für Arbeiten, die dieser Idee eher kritisch gegenüberstehen und die These, dass Atatürk Alevit gewesen sei, ablehnen, siehe Bahadır, 2002: 9-19; Küçük, 2003: 131, 204-207. 
Martin Sökefeld argumentiert, dass Vereine, die das Alevitentum nicht als vom Islam getrennt betrachten, der CEM-Stiftung nahe stehen. Diese These wird jedoch dadurch widerlegt, dass zum Zeitpunkt der Veröffentlichung seines Artikels ${ }^{11}$ keine Aktivitäten der CEM-Stiftung in Deutschland zu verzeichnen waren. Darüber hinaus beschritten die betroffenen Vereine den Weg der Institutionalisierung bereits etwa 10 Jahre vor der Entstehung der CEM-Stiftung. Während der Begriff “Alevitentum”, wie Gümüş betont, in der Gründungserklärung der CEM-Stiftung keine Erwähnung fand (2007: 195) wurde die Pflege des Alevitentums als Hauptzweck in die Satzungen der betroffenen Vereine aufgenommen. Vergleicht man beide Institutionen hinsichtlich ihres Verständnisses vom Alevitentum, lässt sich in der Tat erkennen, wie auch Cemal Salman für das Kölner Beispiel festgestellt hat ${ }^{12}$, dass die Dede-zentrierten Organisationen der Cem-Stiftung insofern ähneln, als dass sie das Alevitentum nicht als vom Islam getrennt betrachten. Von einem institutionellen Schulterschluss kann allerdings nicht die Rede sein. Beide Institutionen verfolgen ihre Aktivitäten in Deutschland unabhängig vom türkischen Kontext. Ein auffallendes Merkmal der Dede-zentrierten Organisationen ist, dass die Gründer und Vertreter der Vereine versuchen, sich aus politischen Konflikten herauszuhalten. Kritik an der türkischen Regierung, wie sie innerhalb kulturzentrierter Organisationen häufig geübt wird, stellt daher in solchen Organisationen mangels einer klar entwickelten politischen Haltung eher die Ausnahme dar (Aksünger, 2013: 142,143; Kehl-Bodrogi, 2006: 9). Vorrangig konzentrieren sich ihre Aktivitäten auf die Stärkung des Glaubens und der Verbundenheit mit dem türkischen Heimatland.

Bevor wir uns nun den Entstehungsprozessen der Organisationen selbst zuwenden, scheint es angebracht, kurz noch einige Informationen über Şinasi Koç anzuführen. Es heißt, dass Şinasi Koç, geboren 1911 im Bezirk Pertek in der Provinz Tunceli, vom achten Imam, Imam Ali er-Rıza (770-818), abstammt. Gemeinsam mit seiner Familie verließ Koç Pertek und ließ sich in Erzurum/Hasankale nieder. Später zog er nach Ankara, wo er den Großteil seines Lebens verbrachte. Şinasi Koç Dede, Vater von sechs Kindern, darunter fünf Jungen und ein Mädchen, widmete sich ein Leben lang dem Dienst am alevitischen Glauben. Bis zu seiner Rente war er in einer

11 Sökefelds Artikel wurde 2015 in der Türkei veröffentlicht. Siehe Sökefeld, 2015: 414.

12 Im Gespräch zwischen Salman und den Verantwortlichen des Kölner Hacı Bektaș Veli Cemevi, dass hier als die erste Dede-zentrierte Organisation betrachtet wird, erklärten die Verantwortlichen, dass sie in Bezug auf das Alevitentum mit der Cem-Stiftung einer Meinung seien. Siehe Salman, 2015: 356. 
staatlichen Einrichtung beschäftigt. Sofern die von Şener und İlknur gemachten Angaben korrekt sind, muss er um 1976-77 zum ersten Mal nach München gekommen sein (1995: 117). Ab den 1980er Jahren war Koç immer häufiger im Ausland unterwegs und spielte vor allem bei der Entstehung alevitischer Vereine in Deutschland eine führende Rolle. Şinasi Koç, der auch bei Cems in Belgien und den Niederlanden als Dede auftrat, unterstützte vor allem die Vereine, die sich um die in Ahlen sitzende Zeitschrift für Wahre Wissenschaft herum organisierten, mit folgendem Ratschlag: "So wie Sunniten in jeder Siedlung ibre Moscheen und Christen ibre Kirchen haben, sollten auch Aleviten einen Ort, einen Verein haben, an dem sie sich versammeln und ibre Cems abbalten können." ${ }^{13}$ Koç setzte sich für eine Neuordnung der alevitischen Rituale und Gottesdienste nach dem gregorianischen Kalender ein und wies die Personen in seinem Umfeld darauf hin, dass das Muharrem-Fasten zwischen dem 27. März und dem 8. April stattfinden sollte, da sich die Schlacht von Kerbela im Frühjahr und nicht, wie angenommen, im Herbst zugetragen habe. ${ }^{14}$ Nach Angaben von Niyazi Bozdoğan behauptete Koç, dass der Koran verändert worden und das unverfälschte Original des Korans in Ägypten zu finden sei ${ }^{15}$, weshalb er sein Umfeld um finanzielle Unterstützung bat, um nach Ägypten reisen und seine Forschungen vertiefen zu können. Jedoch verstarb er am 28. April 1990 in Homburg Saar, ohne diesen Wunsch verwirklicht zu haben. Nach seinem Tod wurde Koçs Leichnam in die Türkei überführt und in Ankara beigesetzt. Zeynel Gül zufolge war Şinasi Koç persönlich an der Gründung der Vereine in Ahlen, Dortmund, Köln, Saarbrücken, Mainz-Wiesbaden, Rheda-Wiedenbrück, Gladbeck und Bergkamen beteiligt (2002: 371).

13 Zitiert von, Gül, 1995: 46; 1999: 85, 86.

14 Für eine kurze Biografie Şinasi Koçs siehe Cevahir, 2014: 181,182. Zudem ist bekannt, dass Koç selbst mehrere Bücher verfasst hat. Im Rahmen dieser Studie konnte er als Autor folgender Werke ermittelt werden: Gerçek İslam Dinini Kimler Bozdu? Kur'an, İncil Tevrat’a göre Gerçek İslam Dini (1970), Gerçek İslam Dini Kur'an ve Ehlibeyt Nedir? (1978); Gerçek İslam Dini Nedir? Kur'ana Bakar mısınız? (1983), Gerçek Kur'an Tarihini Okumak İster misiniz? (1986), und Allah İnsanlardan Ne istiyor? Doğruluk, Teşekkür (1988)

15 Wahrscheinlich hegte er die Hoffnung, unter den Artefakten der Fatimiden, die auch in Ägypten ihre Spuren hinterlassen hatten, geeignete Beweise für seine Behauptung zu finden. Gül sieht einen Widerspruch in Koçs Argumentation, da er seine Behauptung, dass der Koran verändert worden sei, wiederum mit Versen aus dem Koran untermauert habe. Gül, 1999: 86,88; Şahhüseyinoğlu, 2001: 251. 


\subsection{Köln}

Der erste von Aleviten in Deutschland gegründete Verein, in dessen Mittelpunkt das Alevitentum stand und der daher für den Zweck dieser Studie als Dede-zentriert gelten kann, entstand in Köln. Şinasi Koç Dede, der seine Cems nach einem traditionellen Verständnis des Alevitentums leitete, hatte aufgrund seiner aktiven Bemühungen und Zureden großen Anteil an der Gründung des Vereins. ${ }^{16}$ Seinen Anregungen folgend gründeten die Aleviten unter der Führung von Niyazi Bozdoğan, Hüseyin Tur, İbrahim Salper, İsmail Tanriverdi und Turan Ant, der von dem gleichen Ocake kam wie Şinasi Koç, den Verein zur Förderung der Hacı Bektass Veli Kultur in Köln und Umgebung. Der Antrag auf Eintragung des praktisch schon seit 1984 existierenden Vereins (Salman, 2017: 43; 2015: 481, 482). ${ }^{17}$ ins Kölner Vereinsregister wurde am 1. März 1987 genehmigt. Obwohl die offizielle Gründung somit erst 1987 erfolgte, erklärt Niyazi Bozdoğan Dede, ein Mitglied des Aguiçen-Ocaks, in einem im Rahmen dieser Studie durchgeführten Interview, dass erste inoffizielle Treffen und Cems bereits 1984 in Köln stattgefunden hätten. Während eines von Şinasi Koç geleiteten Cems in einem Keller in Köln habe sich Koç über den Platzmangel beschwert und einige Personen, die er für einflussreich und fähig hielt, aufgefordert, in die Dar zu treten, d.h., vor der Gemeinde Rechenschaft abzulegen. So wollte er sie dazu bewegen, ein Versprechen zu geben, dass sie einen Verein gründen würden. Eine dieser Personen sei Niyazi Bozdoğan selbst gewesen. Da er sich darüber im Klaren gewesen sei, dass ein Versprechen, das in der Dar abgelegt wird, erfüllt werden musste, habe er sich zunächst geweigert, in die Dar zu treten. Auf Drängen von Şinasi Koç habe er dies letzten Endes dann doch getan und versprochen, einen Verein zu gründen und einen geräumigen Ort für die Aleviten zu schaffen. ${ }^{18}$

16 Zwar erwähnt Sökefeld diesen in Köln entstandenen Verein nicht, allerdings berichtet er von der Zusammenarbeit zwischen Şinasi Koç Dede und Niyazi Bozdoğan Dede. Siehe Sökefeld, 2002: 171.

17 Ohne einen konkreten Namen oder Ort zu nennen, schreibt İsmail Kaplan in seinem Buch "Das Alevitentum", dass der erste Verein in Europa 1986 gegründet wurde. Wahrscheinlich meint er damit den Verein in Köln. Siehe Kaplan, 2004: 116.

18 Bozdoğan zufolge entfernten sich Koç und der Kölner Verein, an dessen Gründung er aktiv beteiligt war, voneinander, nachdem es in Diskussionen über die Schlacht von Kerbela und den richtigen Zeitraum des Muharram-Fastens, die von der Zeitschrift für Wahre Wissenschaft ausgelöst worden waren, zu Meinungsverschiedenheiten gekommen war. Die Verantwortlichen des Vereins zur Förderung der Hacı Bektaş Veli Kultur in Köln und Umgebung hätten daraufhin beschlossen, unabhängig von Şinasi Koç weiterzuarbeiten. 
Da es zuvor keine andere Organisation gegeben hatte, die das Alevitentum explizit in den Mittelpunkt ihrer Aktivitäten gestellt hätte, hätten Bozdoğan und seine Freunde anfangs nicht so recht gewusst, was sie eigentlich tun sollten, und machten sich daher zunächst Gedanken über die Rechtmäßigkeit des zu gründenden Vereins. Obwohl der Verein in Deutschland gegründet werden sollte, wollte man sich auch eine Genehmigung seitens der Türkei einholen, weshalb die Mitglieder des Gründungsvorstands darauf achteten, dass die von ihnen erarbeitete Satzung sowohl den deutschen als auch den türkischen Gesetzen entsprach. Da alle Gründer türkische Staatsbürgerschaft besaßen, waren viele von ihnen beunruhigt und befürchteten Probleme bei der Einreise in die Türkei, auch wenn der Verein seinen Sitz in Deutschland haben sollte. Deshalb schickten sie die von ihnen vorbereitete Vereinssatzung über das Kölner Konsulat an die zuständigen Behörden in der Türkei und warteten auf deren Genehmigung. Als das türkische Kulturministerium in seinem Antwortschreiben zum Ausdruck brachte, dass der zu gründende Verein an die deutschen Gesetze gebunden sei und der türkische Staat sich deshalb in keinerlei Weise in diese Angelegenheit involvieren würde, leitete man das Gründungsverfahren des Vereins schließlich ein. Trotzdem erwies es sich als schwierig, die in Deutschland zur Gründung eines Vereins nötigen sieben Personen zusammenzuscharen, da die meisten Leute Vorbehalte äußerten und nicht als Gründungsmitglieder auftreten wollten. Schließlich schlossen sich Hüseyin Tur, İsmail Tanrıverdi, İbrahim Salper, Turan Ant, Hüseyin Yağmur, Ali Ergül unter dem Vorsitz von Niyazi Bozdoğan zusammen und reichten den offiziellen Antrag zur Gründung des Vereins zur Förderung der Hacı Bektaş Veli Kultur in Köln und Umgebung e.V. beim Kölner Vereinsregister ein. Der am 1. März 1987 mit der Registernummer 9599 gegründete Verein war die erste offizielle institutionelle Einrichtung, in deren Zentrum das Alevitentum stand. Derweil waren alle Gründer des Vereins als Lohnarbeiter tätig. Dies zeigt, dass sie kein anderes Ziel verfolgten, als den alevitischen Glauben am Leben zu erhalten, und keine weiteren Verbindungen zu anderen Organisationen hatten. In ihrem Versuch, eine Genehmigung von den staatlichen Institutionen der Türkei einzuholen, offenbart sich unterdessen ihre Unerfahrenheit bei der Gründung eines Vereins. Allerdings konnten sie gerade durch diese anfängliche Unerfahrenheit nachfolgenden Dede-zentrierten alevitischen Vereinen helfen, indem sie die Aleviten in anderen Städten, die ebenfalls besorgt waren, dass sie in der Türkei aufgrund einer Vereinsgründung Probleme bekommen könnten, beruhigten und somit zur 
Beschleunigung der dortigen Gründungsprozesse beitrugen (Niyazi Bozdoğan, “Interview vom 08.06.2020"). ${ }^{19}$

\subsection{Mainz}

Soweit dies im Rahmen dieser Studie ermittelt werden konnte, folgten auf den ersten in Köln gegründeten Dede-zentrierten Verein Bestrebungen zur Gründung eines Vereins in Mainz. Angesichts der Tatsache, dass Hüseyin Tur, einer der Gründer des Vereins zur Förderung der Haci Bektas Veli Kultur in Köln und Umgebung, und Derviş Tur, einer der führenden Namen bei der Vereinsgründung in Mainz, Brüder sind, erscheint dies wenig verwunderlich. Sowohl im Hinblick auf seinen Gründungsprozess als auch auf die Rolle, die seine Gründer in der späteren alevitischen Bewegung einnehmen sollten, ist eine Beschäftigung mit dem Mainzer Verein durchaus lohnenswert. Am 26. März 1987 organisierte eine Gruppe von Aleviten, darunter Derviş Tur, Ahmet Aydemir, İsmail Yağlı und Veli Ocaklığlu, eine große Versammlung. Zu dieser Versammlung, an der etwa 50 Personen aus Mainz und Umgebung teilnahmen, wurde auch Hüseyin Tur eingeladen, um über die Erfahrungen zu berichten, die er bei der Gründung des Kölner Vereins gemacht hatte. Obwohl die Versammlungsteilnehmer allesamt überaus gewillt waren, dem alevitischen Glauben zu dienen, herrschte dennoch Unschlüssigkeit, was die Gründung eines Vereins betraf. Hauptsächlicher Anlass hierfür war, ähnlich wie zuvor schon in Köln, die Sorge, dass es bei Reisen in die Türkei zu Problemen kommen könnte, sollten sie Mitglied oder gar in der Führung eines alevitischen Vereins sein. Als diese Bedenken in der Sitzung zur Sprache kamen, ergriff Hüseyin Tur das Wort und erklärte, dass sie zuvor in Köln mit Unterstützung von Şinasi Koç den Verein zur Förderung der Hacı Bektaş Veli Kultur gegründet und sich während des Gründungsprozesses an das Kulturministerium der Türkei gewandt hätten. Er teilte den Anwesenden mit, dass das Kulturministerium der Türkei den Kölner Antrag folgendermaßen beantwortet hatte: "In die Angelegenheiten einer in Deutschland gegründeten und nach deutschem Recht legalen Institution wird keinerlei Eingriff seitens der Türkischen Republike erfolgen.” Derviş Tur sagt, dass diese Erläuterungen seines Bruders die Bedenken der Teilnehmer des Treffens weitgehend zerstreuten, so dass in der Folge einstimmig beschlossen wurde, die Mainz Bektaschi-Gemeinde e.V. zu gründen (“Interview vom 13.12.2017”; 2016: 10).

19 Siehe außerdem Salman, 2015: 482-484. 
Nachdem man auf der Versammlung vom 26. März 1987 beschlossen hatte, einen Verein zu gründen, fand am 27. September 1987 die Gründungsversammlung statt, auf der unter anderem die Satzung des Vereins ausgebarbeitet wurde. Unter dem Vorsitz von İsmail Yağlı gründeten die anwesenden Gründungsmitglieder, unter denen sich unter anderem Derviş Tur, Ahmet Aydemir, Veli Ocaklığlu, Durak Albayrak, Musa Bakır, Aliriza Çelebi, Avni Yüksel und Mukaddim Yüksel befanden, auf dieser Versammlung den Verein. Der Antrag auf Eintragung des Vereins im Vereinsregister wurde nach Fertigstellung der Satzung am 28. Februar 1988 beim Amtsgericht Mainz eingereicht. Bezüglich des Zwecks des Vereins werden in der Satzung folgende Aspekte benannt: "Der Verein setzt sich für die Förderung und Verbreitung des alevitisch-bektaschischen Glaubens und der Kultur... die Eröffnung von Gotteshäusern und Bibliotheken ein... verfolgt keine politischen Zwecke." (Satzung der Mainz Bektaschi-Gemeinde e.V.: 1) Die offizielle Gründung des Vereins wurde dadurch hinausgezögert, dass die Satzung erst noch ins Deutsche übersetzte werden musste und der Sachbearbeiter im Mainzer Vereinsregister sich nicht mit dem Alevitentum auskannte. Als jedoch endlich alle formellen Voraussetzungen erfüllt und auch sonst alle weiteren Probleme gelöst waren, wurde die Mainz. Bektaschi-Gemeinde e.V. am 1. Dezember 1988 mit der Registernummer 2423 als selbständiger Verein ins Vereinsregister eingetragen. Kurz darauf beschloss der Mainzer Verein, eine Veranstaltung zur "Geschichte des Alevitentums" auszurichten, die am 5. Februar 1989 im Astheimer Bürgerhaus stattfinden sollte. Nach Ankündigung der Veranstaltung gingen Drohungen von linken/marxistischen Gruppierungen ein, die den Veranstaltungsort vorgeblich bombardieren wollten. Das Organisationskomitee ließ sich jedoch nicht von seiner Entscheidung abbringen und suchte nach Maßnahmen, um unerwünschte Vorkommnisse zu verhindern. Derviş Tur entschloss sich, die Brüder İbrahim Derin und Harabi Derin, die in Rüsselsheim fernöstliche Sportarten unterrichten, um Hilfe zu bitten. Am Veranstaltungstag sorgten die Derin-Brüder gemeinsam mit ihren Schülern für die Sicherheit im Saal des Bürgerhauses und letztlich gab es auch keine negativen Vorkommnisse zu vermelden $(2016: 18,24) .^{20}$

20 Ähnliche Szenarien gab es in zahlreichen deutschen Städten. So berichtet beispielsweise Ruth Mandel, dass linke Gruppen Anfang der 1990er Jahre in Berlin versuchten, einen Cem zu sabotieren. Siehe Mandel, 2000: 63. 


\subsection{Die Zeitschrift für Wahre Wissenschaft}

Zeynel Gül berichtet, dass er während eines Heidelberg-Besuches anlässlich einer "Y1lmaz-Güney-Gedenknacht" an einem Bücherstand herumstöberte, als er einen Dede sah, der die Zeitschrift, die er in der Hand hielt, in der Luft schwenkte und rief: "Das Alevitentum wird nicht in Vergessenheit geraten!... Dazu reicht eure Kraft nicht aus?"21 Gül erzählt weiter, dass sich dieser Dede daraufhin eine hitzige Diskussion mit dem Besitzer des Bücherstandes, an den er auf die Zeitschrift gestoßen war, geliefert habe (2002: 372; 1999: 85). Bei diesem Dede handelt es sich um Kamber Kutlu, der der Redaktion der Zeitschrift für Wahre Wissenschaft angehörte. Kutlu war außerdem Teil der Gruppe, die auf Ermutigung von Şinasi Koç Dede ihre Arbeit aufnahm. Wie zuvor in Köln, spielte Şinasi Koç Dede eine wichtige Rolle bei der Gründung der Vereine in Ahlen, Aachen, Lübeck und vor allem Bochum, dem Wohnort Kamber Kutlus. Als Zentrum der Zeitschrift für Wabre Wissenschaft sticht unter diesen Vereinen der Alevitische Kulturverein Ablen und Umgebung heraus. Die Vorbereitungen zur Gründung des Vereins wurden 1987 aufgenommen, bevor im Februar 1988 die offizielle Gründung unter dem Vorsitz von Muhsin Cevahir erfolgte. (Ablener Volkszeitung, "Türkische Aleviten mit Fest in der F.-Ebert-Halle", 18. Januar 1988) Die alevitischen Vereine rund um die Zeitschrift für Wahre Wissenschaft sind dem türkischen Staat mit absoluter Loyalität verbunden und legen Wert darauf, bei ihren Cems unbedingt eine türkische Flagge und Porträts der 12 Imame sowie insbesondere auch ein Porträt von Atatürk aufzuhängen. Mehmet Caba zufolge zeigten sich nach der Gründung des Ahlener Vereins auch die in den umliegenden Städten, insbesondere in Kamen und Bergkamen lebenden Aleviten bestrebt, sich formal zu organisieren. Caba behauptet, dass der Vorsitzende des Ahlener Vereins, Muhsin Cevahir, versucht habe, die in Bergkamen lebenden Aleviten zu Mitgliedern des in Ahlen gegründeten Vereins zu machen, aber dass diese stattdessen am 15. Oktober 1989 in ihrer eigenen Stadt die Alevitische Gemeinde Kamen-Bergkamen gründeten (2014: 182-185). Laut der Ausgabe der Zeitschrift Wahre Wissenschaft aus dem September 1994 tummelten sich folgende Vereine im Umfeld der Zeitschrift: der Alevitische Kulturverein Aachen und Umgebung, der Alevitische Kulturverein Ahlen und Umgebung, der Alevitische

21 Obwohl Gül lediglich den Namen der betroffenen Zeitschrift nennt, kann davon ausgegangen werden, dass es das Cover der am 27. September 1987 erschienenen 38. Ausgabe der Zeitschrift Nokta war, dass Kamber Kutlu Dede aufschreien ließ. Siehe: "Alevilik Tarihe Karışıyor: Dinsel, Kültürel ve politik bir yok oluşun öyküsü...”, Nokta 5/38 (27. September 1987). 
Hacı Bektaş Veli Kulturverein Augsburg, der Alevitische Verein Bochum und Umgebung, die Türkische Hacı Bektaş Veli Kulturgemeinde Lübeck und Umgebung, der Alevitische Kulturverein Radolfzell, der Alevitische Kulturverein Selb und der Alevitisch-Bektaschitische Kulturverein Voralberg aus Österreich (Gerçek İlim,1994: 39). Obwohl Şinasi Koç abweichende Meinungen in Bezug auf den Muharrem und das Opfer verteidigte, werden er selbst und die Vereine des um seine Person herum entstandenen Wahre-Wissenschafts-Kreises im Rahmen dieser Studie aufgrund ihrer großen Sensibilität für das Heilige als Dede-zentriert angesehen.

\subsection{Duisburg}

Eine weitere Institution, die den Dede-zentrierten Organisationen zuzurechnen ist, ist der Hünkar Hacı Bektaş Veli Kultur- und Förderverein, der am 28. Mai 1987 in Duisburg gegründet wurde. Es dauerte bis zum 13. Dezember 1988, bis der Verein offiziell in das Vereinsregister eingetragen wurde. Sein erstes Mitglied war Ali Yakar und sein Gründungsvorsitzender Zeynel Demir ${ }^{22}$ vom Derviş-CemalOcak. Zu den vorrangigen Gründungszwecken und -zielen des Vereins gehörte, das friedliche Zusammenleben seiner Mitglieder mit Menschen unterschiedlicher Konfession und kultureller oder ethnischer Zugehörigkeit zu gewährleisten, die sozialen, kulturellen und religiösen Bedürfnisse der Mitglieder zu befriedigen und sie bei der Integration in die deutsche Gesellschaft zu unterstützen, sowie den alevitisch-bektaschitischen Glauben an die Folgegenerationen weiterzugeben und dabei im Einklang mit den gesellschaftlichen Grundbedingungen säkulare und demokratische Prinzipien zu achten. Duisburg ist eine der Städte, die sich durch eine besonders große türkische Bevölkerung auszeichnen. Laut Aksünger beläuft sich diese in der Stadt auf etwa 54.000 Personen. Aksünger führt dabei an, dass es innerhalb dieser türkischen Bevölkerung 10-15 Tausend Aleviten gebe, deren Mehrheit ursprünglich aus der Stadt Erzincan komme, die weiterhin allerdings zu großen Teilen auch aus den Städten Sivas, Tunceli und Çorum stammten. Nach den Gewaltereignissen in Sivas im Jahre 1993 wuchs das Interesser alevtischer Bürgerinnen und Bürger an dem Hacı Bektaş Veli Kultur- und Förderverein immens. Platzmangel und die schwierige Erreichbarkeit des bestehenden Vereins machten die Gründung einer neuen alevitischen Institution in Duisburg erforderlich, was denn 1994 mit der Gründung des Alevi-Bektaschi Kulturvereins im Duisburger Stadtteil

22 Kemal Demir, Hünkar Bingöl und Garip Eken, die drei Vereinsvorsitzenden nach Zeynel Demir, waren ebenfalls Ocakziades. 
Marxloh auch geschah (2013a: 49). Infolge dieser Spaltung benannte der Hacı Bektaş Veli Kultur- und Förderverein sich durch einen auf der Mitgliederversammlung vom 5. Februar 1995 gefassten Beschluss in die Alevitische Gemeinde Duisburg um. (Aksünger, 2013a: 148-151; Alevilerin Sesi 29 (Oktober 1998), 48; Kaçmaz, 2018: 326; Yaşar und Korkmaz, 2009: 77, 159). Die Alevitische Gemeinde Duisburg, die von zwei gleichberechtigten Vorsitzenden geleitet wird, macht vor allem durch seine auf alevitische Jugendliche zugeschnittene Bildungsarbeit unter der Koordination von Bülent Korkmaz auf sich aufmerksam. Besonders nennenswert ist dabei das Kinderbuch “Die Bewohner von Rıza Şehri” (Korkmaz, 2017)23, das geschrieben wurde, um Kindern im Grundschulalter alevitische Werte nahezubringen. Im Rahmen der Feierlichkeiten zu ihrem dreißigjährigen Bestehen organisierte die Gemeinde vom 11.-15. Dezember 2019 Podiumsdiskussionen und kulturelle Veranstaltungen, an denen zahlreiche Wissenschaftler und Künstler teilnahmen (Yaşar und Korkmaz, 2009: 261). Die Alevitische Gemeinde Duisburg befand sich außerdem unter den Gründern der 1991 offiziell gegründeten Föderation der Alevitischen Gemeinden.

Freilich können im Rahmen dieses Artikels nicht die Gründungsgeschichten aller Vereine, die 1991 an der Gründung der Föderation der Alevitischen Gemeinden beteiligt waren, dargestellt werden. Doch es sollte zumindest gesagt sein, dass auch diese Vereine, zu denen unter anderem der Türkische Kulturverein Dortmund, der Hacı Bektaş Veli Kulturförderverein Köln und Umgebung e.V., der Hacı Bektaş Veli Kultur- und Förderverein Duisburg, der Bektaschi-Kulturverein Heilbron, die Bektaschi-Gemeinde Mainz, das Bektaschi-Kulturhaus Stadtallendorf, das BektaschKulturzentrum Frankfurt, das Bektasch-Zentrum Kassel, der Bektaschi-Verein Herzogenburg und der Bektaschi-Kulturverein Filderstadt (Aydoğdu und Aydemir, 2013; Tur, 2016: 38-42). gehören, als Dede-zentrierte alevitische Institutionen betrachtet werden dürfen. Die Anzahl der Dede-zentrierten Vereine ist mittlerweile zu groß, als dass sie sich genau ermitteln ließe. Während sich viele dieser Vereine unter dem Dach der $\mathrm{AABF}$ zusammengeschlossen haben, ziehen einige es vor, unabhängig zu agieren. Dabei war in der Vergangenheit zu beobachten, dass die führenden Personalien der Dede-zentrierten Vereine bis zu den Gewaltereignissen von Sivas am 2. Juli 1993 in der Föderation der Alevitischen Gemeinden, die 1992 in die Föderation Alevitischer Gemeinden in Deutschland (AABF) umbenannt wurde, dominierten. In der Annahme, dass sie die nach den Gewaltereignissen von Sivas

23 Siehe außerdem Yaşar und Korkmaz, 2018: 7, 8. 
zunehmenden politischen Forderungen der Aleviten nicht länger würden bedienen können, überließen sie die AABF-Führung Persönlichkeiten aus kulturzentrierten Institutionen, die sie für politisch fähiger hielten.

\section{Kulturzentrierte Institutionalisierung}

Kulturzentrierte Strukturen, deren erstes Beispiel in Hamburg ausgemacht werden kann, bildeten sich zunächst vor dem Hintergrund des Ziels heraus, die alevitische Kultur zu fördern, da ihre Mitglieder das Alevitentum als genau das, als Kultur betrachteten. ${ }^{24}$ Von positiven Reaktionen ermutigt fasste man mit der Zeit den Entschluss, sich zu institutionalisieren, und so entstanden aus den anfänglichen informellen Strukturen Vereine, in denen auch der Glaube praktiziert wurde. Massicard hebt hervor, dass das Hauptziel von Kreisen, die das Alevitentum als kulturelle Identität betrachteten, darin bestand, das Alevitentum jenseits ethnischer Konflikte zwischen beispielsweise türkischen und kurdischen Aleviten als universelle Identität zu etablieren oder aber ethnische Marginalisierungen innerhalb des anatolischen Alevitentums zu verhindern (2007: 231). In dieser Phase versucht man, die offenen und zukunftsorientierten Elemente der alevitischen Kultur mit den Anforderungen der multikulturellen Gesellschaft zu verweben (Kula, 2012: 173). Im Gegensatz zu den Dede-zentrierten Organisationen ging es bei der Institutionalisierung nicht allein darum, das Bedürfnis nach einem Ort zu stillen, an dem der Glaube praktiziert werden konnte, sondern eben auch eine Einrichtung zu schaffen, die ein Zentrum des sozialen und kulturellen Austauschs sein würde. Diese Präferenz lässt sich gegebenenfalls dadurch erklären, dass, wie auch Sökefeld betont, an der Gründung dieser Vereine kaum Ocakzades - im Falle des Hamburger Vereins tatsächlich nicht ein einziger - beteiligt waren und die Mehrheit der Gründer sich in ihrer Vergangenheit in linken/marxistischen Organisationen politisch engagiert hatten (Sökefeld, 2002: 171, 172; Sökefeld, 2008a: 202; Aksoy und Tekten Aksürmelï, 2018: 90; Massicard, 2007: 78, 79). Dementsprechend betraf die grundlegendste Veränderung in solchen Organisationen, wie auch Langer anmerkt, die Rolle, die man den Dedes zuwies. Gelten die Dedes in Dede-zentrierten Organisationen als die anführende und in Bezug auf die traditionellen Rituale ausführende Figur, werden sie in kulturzentrierten Organisationen auf den Status eines Arbeitnehmers reduziert (Langer, 2015: 493, 496). Soweit ersichtlich, werden die Dedes, die im traditionellen

24 Ali Rıza Gülçiçek, einer der Gründer des Kölner AKM, gibt an, dass man das Alevitentum in jenen Jahren als Kultur betrachtet habe. Siehe Gülçiçek, 2018: 69. 
Alevitentums für interne Einheit Sorge tragen, von dem Verein als wichtigsten Träger der institutionellen Identität abgelöst. Es wird allgemeinhin als eine Idee der kulturzentrierten Organisationen betrachtet, das Alevitentum und seine Rituale zu standardisieren und die in sämtlichen Vereinen abgehaltenen Cems einander anzugleichen (Kehl Bodrogi, 1996: 62). Hierdurch wurde die institutionelle Identität über den Dede als Repräsentanten der Tradition gestellt, was in der Konsequenz dazu führte, dass die heilige Autorität der Dedes innerhalb der Vereine einen Rückschlag erlitt. In einigen Vereinen gibt es auch Personen, die keine Ocakzade sind, aber Cems mit als Babas leiten, zu denen sie von Veliyettin Hürrem Ulusoy, dem Postnişin des Hacı Bektaş Veli Derwisch-Ordens, autorisiert worden sind. ${ }^{25}$

In kulturzentrierten Organisationen stehen wie gesagt die kulturellen Werte des Alevitentums meist im Vordergrund. Allerdings werden diese mit Elementen linker Ideologie vermischt und der Gesellschaft so als Alevitentum präsentiert. In dieser Hinsicht kann man in ihrem Fall von einer reformistischen Orientierung sprechen. Man ist der Auffassung, dass das Alevitentum, wenn es auch in der Zukunft Bestand haben soll, umfassende Reformen durchlaufen müsse, da sich die Lebensbedingungen im Vergleich zu früheren Zeiten stark verändert hätten (Kehl Bodrogi, 1996: 57). Im Zuge einer Einspeisung der religiösen Bestandteile der alevitischen Kultur in die westliche Kultur wurden unter anderem auch neue Rituale entwickelt. Bei der Durchführung dieser Rituale werden westliche Akzente gesetzt und es wird versucht, das Alevitentum inhaltlich so zu präsentieren, dass sich 'westliche' Personen nicht an ihm stören. ${ }^{26}$ In kulturzentrierten Organisationen werden auch Cems in deutscher

25 Dazu zählen z.B. Sedat Bican, der im Lauda-Königshofener AKM als Baba fungierte, und Hüseyin Firat, der in der gleichen Rolle im AKM Esslingen auftrat. Hüseyin Firat war zudem Mitglied des im April 2018 gewählten 12-köpfigen Glaubensrates des AABF.

26 Ein Beispiel dafür ist in der Dezemberausgabe 1997 der Zeitschrift Alevilerin Sesi beschrieben. Der alevitische Dede Zeynel Arslan nahm an der kirchlichen Trauung eines alevitischen Mannes und einer christlichen Frau teil und vollzog den religiösen Akt gemeinsam mit dem christlichen Priester. Während des Gottesdienstes führte der Dede sämtlich religiöse Akte in deutscher Sprache durch. Siehe Arslan, 1997: 37-39. Siehe außerdem Gümüş, 2007: 185, 186; Langer, 2015: 484. Auf dem Traditional and New Alevi Institutions Symposium, das am 26.26. Januar 2018 an der Ruhr-Universität Bochum stattfand, zeigte Robert Langer im Rahmen seines Vortrags "Alevitische Rituale im transnationalen Kontext: The Transfer(ences) of Religious Practice between Turkish Metropolis, Western Diaspora, and Anatolian Village" ein Bild, auf dem ein Dede als Weihnachtsmann verkleidet Geschenke an Kinder verteilt. Langer nannte die Stadt Worms als Aufnahmeort des Bildes, das seiner Meinung nach während eines Nikolaus-Cems entstanden sei. Leider scheint ihm die von den Aleviten in der Region Tunceli 
Sprache abgehalten. Dies gilt zum Beispiel für den Cem der Berliner AAKM am 6. November 1999. ${ }^{27}$ Gümüş berichtet, dass die Dedes, die dem traditionellen Alevitentum anhängen, solch neue Praktiken ablehnen und kritisieren (2007: 186187). In den kulturzentrierten Vereien wird oft betont, dass das Alevitentum eine eigene Glaubensrichtung ist, und versucht, eine Distanz zu sunnitischen Muslimen zu wahren. Nach den Ergebnissen der von Sökefeld durchgeführten Umfrage in Hamburg und Umgebung, also an dem Ort, wo sich die ersten kulturzentrierten Organisationen herausbildeten, gaben 37\% der Aleviten an, dass sie das Alevitentum als eine nicht-islamische Glaubensrichtung ansahen (2008a: 201). Ebenso legt man in diesen Vereinen, in denen sich insbesondere Personen aus dem linken Spektrum betätigen, meist eine distanzierte Haltung gegenüber dem türkischen Staat und den Institutionen, von denen man glaubt, dass sie diesen repräsentierten, an den Tag und bemüht sich, Türkei-Flaggen und Atatürk-Porträts so weit wie möglich aus den eigenen Räumlichkeiten rauszuhalten (Kehl Bodrogi, 2006: 9; Sökefeld, 2015: 414.) ${ }^{28}$

Man kann die kulturzentrierten Organisationen bezüglich ihrer politischen Orientierung weiter in zwei Gruppen unterteilen. Während sich im Verhältnis zu Deutschland keine nennenswerten politischen Unterschiede zwischen den Organisationen erkennen lassen, tritt dieser besonders dort zutage, wo es um die Türkei geht. Er liegt nämlich vor allem in der politischen Haltung gegenüber dem türkischen Staat. Gümüş teilt die Organisationen in Deutschland nach ihrer

praktizierte Gağand-Tradition nicht bekannt zu sein. Die Verantwortlichen des Wormser Cemevis, die zu diesem Thema befragt wurden, gaben an, dass es bei ihnen keinen „NikolausCem" gäbe, dass aber die für die Kinder der Gemeinde veranstaltete Gağand-Cem, die kurz vor Weihnachten, von Langer fälschlicherweise als "Nikolaus-Cem" wahrgenommen worden sein könnte. So betrachtet, kann das Tragen eines Weihnachtsmannkostüms durchaus als eine Veränderung angesehen werden, aber die Gağan-Tradition als Nikolaus-Cem zu begreifen, scheint unangemessen. Cems speziell für Kinder zu abzuhalten ist ein neues Ritual in der alevitischen Tradition, das sich gut in die westliche Tradition einpasst. Zum "Nikolaus-Cem" siehe außerdem Langer, "2010: 386; Langer, 2006: 139. Zur Gağand-Tradition, mit der sich auch Mélikoff befasst hat, siehe Mélikoff, 2006: 81. Gümüş hält fest, dass Alevit*innen aus Tunceli die zeitliche Nähe von Gağand und Weihnachten als Gelegenheit sehen, Freundschaft mit den Deutschen zu schließen. Siehe Gümüş, 2007:182,183.

27 Dieser Cem, den Massicard als eine reformistische Show bewertet, fand zur Einweihung eines neuen Cemevi in einem Gebäude statt, dass die AAKM Berlin zu diesem Zweck gekauft und restaurieren lassen hatte. Siehe Massicard, 2007: 337.

28 Es sollte an dieser Stelle jedoch gesagt sein, dass hier ein enger Zusammenhang mit der politischen Haltung der türkischen Regierungen besteht. Betrifft doch die Kritik der Vereine in der Regel die Diskurse politischer Verantwortungsträger in der Türkei. 
politischen Haltung in drei Gruppen ein, wobei er die hier als Dede-zentriert bezeichneten Institutionen als staatsloya ${ }^{2}$, die hier als kulturzentriert bezeichneten Institutionen hingegen aufgrund ihrer unterschiedlichen Haltung gegenüber der Türkei als entweder staatskritisch ${ }^{30}$ oder staatsfeindlich ${ }^{31}$ definiert (2007: 189). Während sich die staatskritischen Organisationen größtenteils unter dem Dach der AABF sammeln, ist die Mehrheit der staatsfeindlichen Organisationen in der Demokratischen Alevitischen Föderation (FEDA) vertreten, der eine Nähe zur PKK nachgesagt wird.

Bei der Festlegung der Tätigkeitsfelder der kulturzentrierten Organisationen kommt ein linker Reflex zum Tragen, weshalb man einen stärkeren Fokus auf gesellschaftliche Tragödien legt, die sich in der Vergangenheit zugetragen haben. Die Veranstaltungen der Organisationen thematisieren unter anderem Dersim, Maraş, Çorum, Sivas, Gazi und Gezi, Ortsnamen, die symbolisch für Gewalterfahrungen stehen, die tiefe Wunden im kollektiven Gedächtnis der Aleviten hinterlassen haben. ${ }^{32}$ Für die kulturzentrierten Organisation gilt daher, was Yıldırım in Form einer pointierten Charakterisierung folgendermaßen beschreibt: " $\mathrm{Zu}$ begeistern ist man für Themen wie das Gedenken an das Madimak-Massaker, den Bau von Moscheen in alevitischen Dörfern, die Abschaffung des Präsidiums für religiöse Angelegenheiten und den obligatorischen Religionsunterrichts und der Status der Cemevis" (2012: 143). Wenngleich das Hauptanliegen darin besteht, das soziale Gedächtnis lebendig zu halten und die Aleviten um das Alevitentum herum zu vereinigen, ist der Umstand, dass man vorwiegend tragische Ereignisse in den Mittelpunkt von Veranstaltungen stellt, zu einem großen Teil auf die politischen Entscheidungen derjenigen zurückzuführen, die an der Gründung der Institutionen beteiligt oder zu deren Wortführer auserkoren wurden. Angesichts der Tatsache, dass ihre Mitglieder sich um begrenzte Ziele herum organisieren und in Abgrenzung zur Religion ihre säkularen Identitäten in den Vordergrund stellen, können die kulturzentrierten Organisationen als ein Beispiel für Kollektive gelten, die dem Tönniesschen Gesellschaftsbegriff entsprechen (Akyüz, 2007: 21, 22). Das Säkularitätsverständnis solcher Einrichtungen spiegelt sich auch in den von ihnen organisierten Veranstaltungen wider. Eher als Dedes werden zu diesen

29 Diese Organisationen sind dem türkischen Staat gegenüber loyal.

30 Diese Organisationen akzeptieren den türkischen Staat, kritisieren ihn jedoch in seinen Handlungen.

31 Diese Organisationen lehnen den türkischen Staat per se ab.

32 Siehe Ataman, 2019: 31. Für eine ähnliche Beobachtung in Bezug auf alevitische Vereine in der Türkei siehe Ceylan, 2015: 217. 
intellektuelle, für ihre säkulare Einstellung bekannte Redner eingeladen (Massicard, 2007: 169), um ihre Meinungen und Vorschläge zu aktuellen Themen zu äußern. Die kulturzentrierten Organisationen, als deren erstes Beispiel das 1989 im Anschluss an die Hamburger Alevitische Kulturwoche gegründete Hamburger Alevitische Kulturzentrum gelten darf, bilden trotz diverser interner Spaltungen ${ }^{33}$ derzeit den Kern der Institutionalisierung von Aleviten in Europa.

Sökefeld, der die Alevitische Kulturwoche in Hamburg als Anfang der alevitischen Institutionalisierung in Deutschland sieht, begründet diese Ansicht damit, dass es sich bei der Kulturwoche um die erste öffentliche Veranstaltung in Bezug auf das Alevitentum in Deutschland gehandelt habe (Sökefeld, 2015: 413; 2003: 143, 144; 2003a: 14/244). Aus den im Rahmen dieser Studie geführten Interviews ging jedoch hervor, dass schon im Februar 1975 ein eintägiger Cem zum Gedenken an den heiligen Hızır (Y1ldı, "Interview vom 23.01.2019”; Bozdoğan, "Interview vom 08.06.2020”; Keleş, 2020: 400) stattgefunden hatte, bei dem etwa 1000 zugegen gewesen sein sollen, darunter auch Vertretern der katholischen und der evangelischen Kirche und zahlreiche Aleviten aus den umliegenden Landkreisen und Bundesländern. Andererseits ist es erstaunlich, dass Sökefeld in seinen Publikationen zwar einen 1984 in Hamburg abgehaltenen großen Cem erwähnt ${ }^{34}$, der im Hamburg der 1980er nur einer von vielen unter der Leitung von Şinasi Koç Dede stattfindenden Cems war, diesen jedoch nicht mit dem Beginn der alevitischen Bewegung in Verbindung bringt. So ist doch nicht zuletzt auch die Genese der Dede-zentrierten Organisationen in Köln im Jahr 1984 zu verorten. Sökefeld stellt damit, willentlich oder unwillentlich, die von den Dedes in Gang gesetzten Institutionalisierungsprozesse in den Hintergrund. Dies soll andersherum freilich nicht heißen, dass die Alevitische Kulturwoche in Hamburg bedeutungslos wäre. Wie im Folgenden zu sehen sein wird, stellt sie sowohl inhaltlich als auch in ihrer Wirkung einen wichtigen Wendepunkt in der deutschen

33 Mit Spaltung ist an dieser Stelle gemeint, dass einige alevitische Organisationen wie die Kurdisch Alevi-Föderation (KAB) und die Revolutionäre Alevitische Föderation (DAB), die einer linken Tradition entstammen, aus den alevitischen Kulturzentren (AKM) Mitglieder für die von ihnen gegründeten Vereinigungen 'abwerben'. Dies gilt im Übrigen auch für das Führungspersonal. So kommen die meisten Führungskräfte von Organisationen wie der $\mathrm{KAB}$ und der $\mathrm{DAB}$ aus der $\mathrm{AKM}$.

34 In dem 2003 veröffentlichten Artikel "'Wir sind auch da!' Aleviten in Hamburg und der Kampf um Anerkennung" erwähnt Sökefeld einen Cem in Hamburg, an dem rund 1000 Personen teilnahmen, und berichtet, dass dieser von Linken protestiert wurde. Siehe Sökefeld, 2003a: 14/251. Siehe außerdem Sökefeld, 2008a: 202. 
alevitischen Bewegung dar. Darüber hinaus markieren die Hamburger Formationen den Ausgangspunkt der kulturzentrierten Organisationen.

\subsection{Hamburg}

Wie bereits erwähnt, wurde der erste Schritt zur Gründung kulturzentrierter Organisation in Hamburg unternommen. Bis Dezember 1988 hatte es dort keine speziell um das Alevitentum herum organisierte Institution gegeben. Seit ihrer Einwanderung nach Deutschland hatten die in Hamburg lebenden Aleviten daher versucht, ihre Bedürfnisse nach einem sozialen Miteinander in Vereinen zu befriedigen, in denen Gruppen unterschiedlicher linker Couleur tonangebend waren. Unter diesen Vereinen spielte der türkisch-sozialdemokratische Verein HDF, die engen Beziehungen zu der Republikanischen Volkspartei (CHP) in der Türkei unterhielt, bis zum Massaker von Maraş im Dezember 1978 eine wichtige Rolle. Die in Hamburg lebenden Aleviten, die die CHP für die Geschehnisse in Maraş mitverantwortlich machten, traten aus der HDF aus und gründeten mit der Föderation Türkischer Arbeiter für den Frieden einen neuen Verein, der sich in der Folge der Föderation der Patriotischen Vereinigungen (YBF) anschloss. Als die YBF 1983 den Beschluss fasste, sich aufzulösen, wurde der Hamburger Verein noch im selben Jahr wegen Mitgliedermangels aufgelöst und sein Eigentum auf den neu gegründeten Verein "Haus für Alle" übertragen, dessen Schwerpunkt auf interkultureller Arbeit lag (Özbaş, 1999: 41; Tosun, 2002: 33,35,36,76). Einige Stimmen, die Hamburg als den Mittelpunkt alevitischer Institutionalisierung sehen wollen, behaupten jedoch, dass dieser Verein 1989 als Alevitisches Kulturzentrum (AKM) gegründet worden sei (Özbaş, 1999: 10, 48). Diese Behauptung erweist sich jedoch lediglich für die Vereine in München und Berlin als korrekt. Während der Münchner Verein sich ab 1990 Tätigkeiten widmete, die sich speziell auf das Alevitentum bezogen, wurde der Berliner Verein 1991 in das Alevitische Kulturzentrum Berlin umorganisiert, wie auch weiter unten noch zu sehen sein wird. In Hamburg hingegen gab es zwischen 1984 und 1989 keine explizit alevitische Institution.

Die Tatsache, dass sich Aleviten in Hamburg um das Alevitentum herum zusammenschlossen, ist, wie in vielen anderen Städten in Deutschland auch, den Bemühungen Şinasi Koç Dedes zu verdanken. Halis Tosun erzählt, dass 1984 in der Aula der Gesamtschule Mümmelmannsberg ein Cem unter der Leitung von Şinasi Koç Dede stattgefunden habe, an dem 600 Menschen teilnahmen. Während dieses Cems wollte Hasan Kilavuz, der in den Jahren 1996-1998 als Vorsitzender des Hamburger 
AKM und in den 2000er Jahren als Vorsitzender des Dede-Rates fungieren sollte, eine Erklärung zur Kurdenbewegung verlesen, wurde davon jedoch letztendlich von Şinasi Koç Dede abgehalten. In jenen Jahren nahmen außerdem rund 1000 Aleviten an dem von Şinasi Koç Dede geleiteten Cem in Hamburg-Altona teil. Die hohe Teilnehmerzahl erklärt sich daher, dass man im Vorfelde Flugblätter verteilt und die Veranstaltung groß angekündigt hatte. Wie Tosun mitteilt, hätten Anhänger der Kurdenbewegung allerlei Anstrengungen unternommen, diese Cems zu verhindern, da sie der Meinung waren, dass diese eine mögliche Institutionalisierung der Aleviten maßgeblich beeinflussen würde (Tosun, 2002: 81, 92). ${ }^{35}$

Die Institutionalisierung der von Şinasi Koç Dede initiierten Zusammenkünfte in Hamburg nahm am 30. Dezember 1988 Gestalt an, als zwölf Personen ${ }^{36}$ den Grundstein für eine neue Organisation namens Alevitisch-Bektaschitischer Kulturkreis Hamburg legten, um zu verhindern, dass die Kinder alevitischer Familien zu Sunniten würden. Die Mitglieder der Gruppe, zu denen auch Turgut Öker, İsmail Kaplan und Halis Tosun gehörten, beschlossen bei ihren Treffen, zunächst eine Veranstaltung an der Hamburger Universität zu organisieren. Tosun berichtet, dass die Gruppe im Vorfeld der Kulturwoche zunächst eine Podiumsdiskussion

35 Zu der Tatsache, dass Şinasi Koç den in Hamburg abgehaltenen Cem leitete, siehe Sökefeld, 2002: 171.

36 Es soll sich dabei um 12 Personen gehandelt haben, sofern die Person, die das gemeinsame Foto des Treffens schoss, mit einberechnet wird. So wird dem Treffen eine gewisse Mystik eingehaucht und versucht, eine Verbindung mit den 12 Imamen herzustellen. Auch das Datum des Treffens wird unterschiedlich angegeben. Während in dem vom Alevitischen Kulturzentrum Hamburg herausgebrachten Buch Onlarn Öyküsü vom 28. Dezember 1988 die Rede ist, meint Halis Tosun, dass die Gruppe sich am 26. Dezember 1988 getroffen habe. İsmail Kaplan hingegen beharrt auf dem 30.12.1988. An dieser Stelle soll zwecks Übereinstimmung mit vorangehenden Publikationen das von İsmail Kaplan angegebene Datum beibehalten werden. Es soll allerdings darauf hingewiesen sein, dass diese Angabe keine gesicherte Erkenntnis darstellt. Weiterführend siehe Kaplan, 2009: 216; Halis Tosun nennt 11 Personen. Namentlich handelt es sich dabei um Halis Tosun, İsmail Kaplan, Turgut Öker, Rıza Cömert, Musa Şen, Esma Tosun, Hüseyin Dörtyol, Zekiye Dörtyol, Ali Altundağ, Hüseyin Yavuz und Oruç Yağbasan. Siehe Tosun, 2002: 93-111; Özbaş, 1999: 8; Keleş, 2020: 402. Turgut Öker berichtet in einem Interview für die November-Ausgabe 2000 der Zeitschrift Alevilerin Sesi, dass die Organisation in Hamburg mit etwa 10 Personen ihren Anfang genommen habe, deren Namen er allerdings nicht erwähnt. Siehe Alevilerin Sesi 40, 2000: 15. Sökefeld hingegen spricht von zwölf Personen, ohne dabei irgendwelche Namen zu nennen, und fügt an, dass sich unter ihnen keine Ocakzades befunden haben, wohl aber einige Personen, die ihrem Selbstverständnis nach Sozialdemokraten und Atheisten waren. Siehe Sökefeld, 2002: 172; 2003a: 14/252. 
organisierte, an der auch Fuat Bozkurt teilnahm, und dann zum Meinungsaustausch nach Berlin reisten, um mit ehemaligen YBF-Mitstreitern wie Süleyman Cem, Ahmet Kömürcü, Ali Asker Aslan und Ali Haydar Cilasun über ihr weiteres Vorgehen zu beratschlagen. Dabei unterstreicht er, dass während dieses Treffens der Entschluss gefällt wurde, die Kulturwoche zu organisieren, obwohl Ali Haydar Cilasun sich klar gegen diese Idee aussprach (Tosun, 2002: 96), von der übrigens zu vermuten ist, dass sie von dem Symposium ${ }^{37}$ inspiriert war, dass 1986 anlässlich Irene Melikoffs Verabschiedung in den Ruhestand an der Universität Straßburg ausgerichtet worden war. In jedem Falle fand als Ergebnis dieses Entschlusses der im Rahmen eines sechstägigen Programms vom 2. bis 7. Oktober 1989 die "Alevitische Kulturwoche" an der Universität Hamburg statt. Am ersten Tag fanden neben den Eröffnungsreden Gespräche mit Gästen aus der Türkei statt. Das Thema des zweiten Tages, an dem Rıza Zelyut und Çetin Yetkin als Referenten geladen waren, waren der Ursprung des Alevitentums, seine Entwicklung in Anatolien und sein Platz in der türkischen Gesellschaft. Am dritten Tag hielten M. Fuat Bozkurt und Nejat Birdoğan für die deutschen Besucher einen Vortrag in deutscher Sprache über den Ursprung des Alevitentums, den Glauben, die Liebe zu den Menschen, den Gottesdienst und die Musik. Am vierten Tag wurden die Themen des Vortages von Riza Zelyut und Nejat Birdoğan erneut aufgegriffen, dieses Mal allerdings auf Türkisch. Am fünften Tag wurde begleitet von Semah-Gebeten ein Cem abgehalten. Am letzten Tag fanden kulturelle Veranstaltungen und Konzerte statt, an denen verschiedene Künstler mitwirkten (Tosun, 2002: 96-97). Die Kulturwoche weckte größeres Interesse als erwartet, was sich auch in den hohen Besucherzahlen widerspiegelte. In der Folge wurden ähnliche Veranstaltungen auch in anderen deutschen Städten organisiert, z.B., wie unten zu sehen sein wird, in Berlin und Köln. Während der Alevitischen Kulturwoche, die ein breites Echo in der Öffentlichkeit fand und in den Erklärungen der AABF als Beginn der alevitischen Bewegung in Deutschland deklariert wurde, obwohl es vor ihr bereits andere alevitische Organisationen gegeben hatte, wurde

37 Im Rahmen des Internationalen Bektaschismus-Symposiums, das von der Universität Straßburg organisiert wurde, fanden neben wissenschaftlichen Vorträgen auch Veranstaltungen statt, die über die alevitische Kultur informieren sollten. Beispielsweise wurde ein öffentlicher Cem abgehalten, den Hasan Taşkale als Dede leitete und Ali Rıza Aslandoğan als Zakir ("Barde") begleitete. In seinem Buch gibt Ali Rıza Gülçiçek an, dass die Veranstaltung 1987 stattgefunden habe. Siehe Gülçiçek, 2018: 56,57. Der Dankesbrief von Melikoff, der dem Autor dieser Studie von Necati Şahin, der mit den Vorbereitungen für den Cem im Rahmen des Symposiums betraut war, zur Verfügung gestellt wurde, belegt jedoch, dass das Symposium zwischen dem 29. Juni und dem 2. Juli 1986 stattfand. 
zudem das sogenannte "Alevitische Manifest" (Alevi Bildirgesi) veröffentlicht, in der das Alevitentum in der Hoffnung, dass die deutsche Gesellschaft es als Glaubensrichtung anerkennen würde, als "lebendiger Bestandteil des Islams" gefasst wurde. Das Manifest, mit dem das Alevitentum laut Şahin zum ersten Mal in aller Öffentlichkeit auftrat (2002: 123), wurde auch von den türkischen Tageszeitungen Cumburiyet und Hürriyet abgedruckt. ${ }^{38}$

In dem vierseitigen Text des "Manifests", das von der Alevitischen Kulturgruppe Hamburg unterzeichnet war, wurde zunächst festgestellt, dass laut offiziellen Statistiken 98\% der türkischen Bevölkerung Muslime seien, wobei die türkische Republik auch die Aleviten zu den Muslimen zähle und diese sich umgekehrt als Muslime ausgaben. Das Manifest war in 15 Abschnitte gegliedert, deren Überschriften wie folgt lauteten: 1) In der Türkei leben 20 Millionen Aleviten. 2) Die Religionsfreiheit ist ein Menschenrecht. 3) Die Glaubensfreiheit ist in der Verfassung verankert. 4) Das Präsidium für Religionsangelegenheiten vertritt lediglich den sunnitischen Zweig des Islam. 5) Die alevitische Identität wird negiert. 6) Aleviten müssen ihre Identität verbergen. 7) Die Menschenrechte werden verletzt. 8) Die alevitisch-bektaschitische Glaubenslehre steht für Demokratie, Säkularismus und Toleranz. 9) In Deutschland leben 350-400 Tausend Aleviten. 10) Die alevitische Glaubenslehre bereichert die multikulturelle Gesellschaft. 11) Die alevitische Kultur muss am Leben erhalten werden. 12) Die zweite Generation Aleviten in Deutschland muss moralisch unterstützt werden. 13) Bessere Verständigung zwischen Aleviten und Sunniten ist nötig. 14) Deutsche sollten mit der alevitischen Glaubenslehre vertraut sein. 15) In Hamburg bedarf es Veranstaltungen, die über die alevitische Kultur aufklären.

Die Kulturwoche, an der zahlreiche Politiker, Akademiker, Schriftsteller und Künstler aus der Türkei und Europa teilnahmen, zog auch die Aufmerksamkeit der deutschen Medien auf sich, die in diesem Zuge über die Aleviten berichteten (Tosun, 2002: 93, 111; Sökefeld, 2003: 143-145; 2015: 412). Im Rahmen des Veranstaltungsprogramms wurde ein Cem abgehalten ${ }^{39}$ und mehrer Konzerte von

38 Am 6. Mai 1990 erschien das "Alevitische Manifest" in der Zeitung Hürriyet und am 15. Mai 1990 wurde es auf der 15. Seite der Zeitung Cumburiyet abgedruckt. Siehe außerdem Özbaş, 1999: 98-101; “Alevilik Bildirgesi” 1989.

39 Sökefeld hält fest, dass die Organisatoren der Kulturwoche zunächst bei Şinasi Koç angefragt hatten, ob er diesen Cem leiten wolle, sich dann aber für jemand anderes entschieden, da 
bekannten alevitischen Künstlern gespielt. Es heißt, dass über 5000 Aleviten aus verschiedenen Regionen Deutschlands an der Alevitischen Kulturwoche teilnahmen. Das große Interesse an der Alevitischen Kulturwoche sorgte dafür, dass die HABKG am 9.-10. Juni 1990 unter dem Vorsitz von İsmail Kaplan den Verein "Alevitisches Kulturzentrum" gründete. Bemerkenswert ist, dass Ali Haydar Cilasun auf der Gründungsversammlung den Vorsitz innehatte. ${ }^{40}$ İsmail Kaplan behauptet, dass die alevitischen Organisationen, die in der Folge in diversen deutschen Städten gegründet wurden, von dem Hamburger Beispiel inspiriert, sich ebenfalls für "Alevitisches Kulturzentrum" als offiziellen Namen entschieden (2009: 216; Sökefeld, 2008b 23; 2008b: 22-24; Keleş, 2012: 158; Dettling, 2006: 35; Tosun, 2002: 113-117). Die Recherchen im Rahmen dieser Studie weisen jedoch eher darauf hin, dass dieser Name von Organisationen bevorzugt wird, in denen sich mehrheitlich linke Aleviten sammeln. Diese Feststellung wird sowohl von Kehl-Bodrogi als auch von Sökefeld bestätigt (2008c: 33; Kehl-Bodrogi, 2003: 462). Eine weitere Perspektive in diese Diskussion bringt Bozkurt. Demnach nennen viele Aleviten der ersten Generation ihre Vereine "Cemevi”, selbst wenn diese als "Kulturzentrum" gegründet worden waren (Bozkurt, 2015: 83). Darüber hinaus konnte beobachtet werden, dass, wie auch am Beispiel der Berliner und Kölner Vereine noch zu sehen sein wird, diejenigen, die Veranstaltungen wie die Kulturwoche organisierten, dies auch vor dem Hintergrund taten, der Öffentlichkeit auf den Puls zu fühlen, bevor man den Weg der Institutionalisierung einschlug. Solche Veranstaltungen ermöglichten es, das öffentliche Interesse festzustellen sowie auch den Bedarf der ansässigen alevitischen Bevölkerung nach institutionellen Strukturen zu ermitteln. Als dann das große Interesse der Aleviten zeigte, dass ein solcher Bedarf in der Tat bestand, beschloss man, sich unter dem Dach eines Vereins offiziell zusammenzuschließen. Aus dieser Perspektive betrachtet scheint es so, dass das Alevitentum in diesem Zusammenhang eher als Instrument bei der Schaffung institutioneller Strukturen diente. Denn diejenigen, die aktiv an der Organisation der Veranstaltungen mitgewirkt hatten, saßen später auch in der Führung der neu gegründeten Vereine. In den Dede-zentrierten Organisationen war genau das Gegenteil der Fall. Hier wurden

Koç verlangte, dass während des Cems eine Türkei-Flagge und ein Atatürk-Poster aufgehängt werden sollten. Siehe Sökefeld, 2002: 172.

40 Bemerkenswert ist dies deshalb, weil Cilasun, nachdem er eine Zeit lang als Dede in der $\mathrm{AABF}$ tätig gewesen war, mit der Kurdischen Alevi-Föderation zusammenarbeitete und eine kurdisch-nationalistische Haltung an den Tag legte. Für weitere Einzelheiten hierzu siehe; Gülçiçek, 2018: 57-60. 
zunächst institutionelle Strukturen begründet und erst dann große Versammlungen organisiert, in denen das Alevitentum vermittelt wurde. Institutionalisierung stellte daher für die Dede-zentrierten Organisationen ein Mittel zur Pflege und Vermittlung des Alevitentums dar.

Die Tatsache, dass İsmail Kaplan, der gute Beziehungen zur Universität Hamburg unterhielt, Veranstaltungen an der Universität ausrichtete, ohne sich dabei der Zustimmung aller Vorstandsmitglieder zu vergewissern, führte zu Diskrepanzen in der Vereinsführung. Als dann İsmail Aslandoğan Dede auch noch bekundete, dass er die Arbeit des Alevitischen Kulturzentrums für nicht nah genug am Alevitentum hielt (Sökefeld, 2002: 173), musste Kaplan zurücktreten. Eine institutionelle Spaltung wurde somit unausweichlich. Nach dem, was in dem zum 10-jährigen Bestehen der Hamburger AMK verfassten Buch "Onlarn Öyküsü” erzählt wird, war ein weiter Grund für die Spaltung die Versuche des damals im Verband tätigen Turgut Öker, seinen Einfluss innerhalb der Hamburger AKM geltend zu machen. ${ }^{41}$ Der kulturzentrierte Ansatz des AKM und die marginale Rolle, die die religiösen Elemente des Alevitentums spielten, müssen denjenigen, die das traditionelle Alevitentum pflegen wollten, wohl ein Dorn im Auge gewesen sein. Nach dem Rücktritt von İsmail Kaplan formte Mehmet Zülküf Kılıç nämlich auf der außerordentlichen Versammlung 1990 eine Oppositionsliste und kündigte seine Kandidatur für den Vorstand an. Als jedoch die Stimmen, die er erhielt, nicht zu seiner Wahl ausreichten, traten er und 35 weitere Personen aus dem Hamburger AKM aus. Zusammen gründeten sie 1991 einen neuen Verein mit dem Namen "Anatolisch-Alevitischer Kulturverein Hamburg” (HAAK-BIR) ${ }^{42}$. Stellvertretend für die Hamburger Aleviten wurde der Verein HAAK-BIR kurz nach seiner offiziellen Gründung Mitglied der Föderation der Alevitischen Gemeinschaften (ACF) (Özbaş 2002: 62,63,69,103104). Die Hamburger AKM trat der ACF 1993 bei. Die Institutionalisierung der Aleviten in Hamburg nahm in den folgenden Jahren weiter zu: Im Jahre 2002 waren 706 Personen als Mitglieder in insgesamt fünf verschiedenen alevitischen Vereinen

41 Es soll jedoch darauf hingewiesen sein, dass Turgut Öker auf dem Kongress im Oktober 1993 in den Vorstand des Verbandes gewählt wurde. Die behandelte Spaltung hingegen trug sich im Jahr 1990 zu. Zu der Behauptung, dass Turgut Öker die Spaltung verursacht habe, siehe Özbaş 1999: 69.

42 Als sich auch in diesem neuen Verein kulturzentrierte Tendenzen zeigten, verließ İsmail Aslandoğan Dede HAAK-Bir in kurzer Zeit und leistete Pionierarbeit bei der Gründung eines dritten Vereins in Hamburg. Siehe Sökefeld, 2002: 173. 
organisiert (Aksünger, 2013: 139). Sökefeld erwähnt, dass 2003 neun verschiedene alevitische Vereine in der Stadt gegründet wurden, deren alevitische Bevölkerung sich seiner Schätzung nach auf etwa 20.000 Personen beläuft (2003a: 14/253).

\section{Berlin}

Der 1979 in Berlin gegründete Deutsch Türkische Arbeiterbund West-Berlin e.V., der im selben Jahr der Patriotischen Vereinigung (TYB) beigetreten war, blieb unberührt davon, dass nach der Auflösung der TBP 1983 auch die YBF ihre Aktivitäten einstellte, und stand bis 1991 weiter im Dienst seiner Mitglieder. 1991 zeitigte der Einfluss der Alevitischen Kulturwoche, die als Fortsetzung der 1987 angeschobenen Institutionalisierungsprozesse durch Dede-zentrierte Verein in Köln von linken Aleviten, die einen kulturzentrierten Ansatz beherzigten, in Hamburg organisiert worden war, auch in Berlin erste Wirkungen. Anders als in Hamburg bildete sich hier allerdings keine neue Organisation heraus. Stattdessen wurde der bereits bestehende Verein 1991 im Zuge einer Namens- und Satzungsänderung offiziell in das Anatolisch-Alevitische Kulturzentrum Cemevi Berlin umgewandelt. Kehl-Bodrogi führt an, dass die Wahl des Namens als Ergebnis einer Umfrage unter rund 200 Aleviten und aufgrund eines gemeinsamen Beschlusses zustande gekommen sei. Später organisierte die Berliner AAKM eine "Anatolisch-Alevitische Kulturwoche". Die einwöchige Veranstaltung fand vom 26. bis 31. März 1991 im Berliner Haus der Kulturen der Welt statt. In den Podiumsdiskussionen und Foren brachten Experten wie Irene Melikoff, Fuat Bozkurt, Server Tanilli, Atilla Özkırıml, Nejat Birdoğan und İlhan Selçuk den Besucher das Alevitentum nahe. Am Ende der Woche, in der unter anderem auch Deyiş (gesungene Dichtungen) vorgetragen wurden, fand ein großer Cem statt. Die Berliner AAKM setzte die Alevitische Kulturwoche in den Folgejahren ununterbrochen fort. Andererseits sorgte das AAKM Berlin 1992 für eine Premiere, in dem es in Berlin einen frei empfangbaren Fernsehsender namens AL-CANLAR TV gründete (İrfan, 1994: 17). Obwohl der Sender nach den Anschlägen vom 11. September 2001 den Betrieb einstellen musste, weil es gesetzlich verpflichtend wurde, sämtliche Sendungen in deutscher Sprache auszustrahlen und es ihm an deutschsprachigem Personal mangelte, ist er als erster seiner Art dennoch von Bedeutung. ${ }^{43}$ Der Berliner AAKM kaufte 1998 ein altes Kirchengebäude und ist seit 2002 für die Koordination des alevitischen Religionsunterrichts an Berliner Schulen zuständig (Kehl-Bodrogi, 2002: 51; Büyükgöl, (Januar 2005): 42; Şahin, 2002:

43 Für detaillierte Informationen zu AL-CANLAR TV siehe Kosnick, 2008: 112-120. 
139; Massicard, 2007: 303; Dağyeli, 2006: 77; Engin, 2015: 121). ${ }^{44}$ Zudem wurde das erste Zertifikat zur Lehrbefugnis für alevitischen Religionsunterricht nach den von AAKM Berlin organisierten Fortbildungsseminaren (Dikmen, 2005: 43; Yüreklik, 1991; Büyükgöl, April 2005: 43) ausgestellt.

Berlin ist nicht nur Schauplatz so mancher Neuheit in Bezug auf das Alevitentum gewesen, sondern ist laut Zirh die einzige Stadt in Deutschland bzw. sogar in ganz Europa, in der Aleviten stärker konzentriert sind als Sunniten (2015: 359). Auf institutioneller Ebene lässt sich diese Behauptung jedoch nicht in gleicher Weise geltend machen. Tatsächlich wurden in der 2006 von Riem Spielhaus durchgeführten Studie insgesamt 83 von Muslimen für ihre religiösen Bedürfnisse geschaffenen gotteshausähnliche Einrichtungen ausgemacht, unter denen aber als einer der zwei großen alevitischen Einrichtungen nur der Berliner AAKM vertreten war (2006: 12-17). Die übrigen acht von Massicard erwähnten alevitischen Vereine ${ }^{45}$ können, wenngleich sie von Aleviten gegründet wurden, als Beispiele für Organisationen gelten, für deren Arbeit die gemeinsame ideologische Orientierung und das Band zwischen den Mitgliedern aufgrund ihrer Herkunft aus den gleichen Gegenden in der Türkei ausschlaggebend sind. ${ }^{46}$ Aus den Erzählungen Tosuns geht hervor, dass Şinasi Koç Dede auch bei der Organisation der Aleviten in Berlin eine wichtige Rolle spielte. Denn vor dem Cem in Hamburg-Altona, an dem 1000 Menschen teilnahmen, leitete Koç einen Cem in Berlin, nach dem er mit Tosun telefonierte und dann von Berlin nach Hamburg fuhr, um an dem dortigen Cem teilzunehmen (2002: 91).

44 Die Mitglieder des Vereins behaupten, dass sie der älteste alevitische Verein in Deutschland seien, da sie ihre Aktivitäten seit 1979 fortführten. Wie jedoch bereits erläutert, bestand der Hauptzweck der Föderation der Patriotischen Vereinigungen und der ihr angeschlossenen Vereine nicht im Alevitentum, sondern neben sozialen Aktivitäten in der Unterstützung der türkischen Einheitspartei. Zum Kauf des Kirchengebäudes, siehe auch Langer, 2010: 492. Alevilerin Sesi 29, 1998: 50.

45 Massicard bemerkt, dass die alevitischen Vereine, deren Anzahl ihren Nachforschungen zufolge bis Ende der 1990er Jahre zehn erreicht hatte, die Idee diskutierten, eine Föderation für das Land Berlin zu gründen. Diese kam aber aufgrund der ideologischen Differenzen zwischen den Vereinen nicht zustande. Siehe Massicard, 2007: 306, 307.

$46 \mathrm{Zu}$ diesen Vereinen gehören beispielsweise Dersim Kulturgemeinde Berlin e.V., Bahadınlı Solidaritätsverein Sivaslı Canlar e.V., Verein zeitgenössischer Demokraten in Tokat und Umgebung e. V., Çorum-Der e.V., BSV Al-Dersimspor e.V. Siehe auch Dağyeli, "Die Aleviten", 76,77; Kehl-Bodrogi, 2002: 58-62. 
Offiziellen Angaben zufolge lebten im Jahr 2005212.723 Muslime in Berlin, während die Zahl der Türken, einschließlich derjenigen, die die deutsche Staatsbürgerschaft trugen, auf etwa 170.000 geschätzt wurde (Kapphan, 2006: 118, 119). $1997 \mathrm{kam}$ es in Berlin, wo angeblich 40.000 bis 50.000 Aleviten leben sollen (Dağyeli, 2007: 75; Kehl-Bodrogi, 2002: 44), zu einer großen Spaltung, im Zuge derer die Hacı Bektaş Veli Kulturgemeinde (HBKC) als neuer Verein gegründet wurde. Kurz nach der Gründung der HBKC nahm Kırk Budak TV, eine Alternative zum Sender AL-CANLAR TV, der religiöse Inhalte für Aleviten ausstrahlte, seinen Sendebetrieb auf. Kurk Budak TV ereilte jedoch dasselbe Schicksal wie AL-CANLAR TV: 2001 musste der Sendebetrieb eingestellt werden. ${ }^{47}$ Nach den Informationen, die Massicard auf Basis der von ihr durchgeführten Interviews vorträgt, lagen der Spaltung in Berlin religiöse und politische Differenzen zugrunde, die an die Situation in Hamburg erinnern. Diejenigen, die der AAKM den Rücken kehrten und die HBKC gründeten, warfen den dort Verbleibenden vor, politischen Themen zu viel Raum zu geben, und bekundeten, dass sie sich stärker dem Glauben widmen wollten. Dementsprechend hielt die Berliner AAKM in ihrer Arbeit an einem kulturzentrierten Ansatz und der Kooperation mit linken Gruppierungen fest, während die HBKC sich, einem Dede-zentrierten Ansatz folgend, von einer traditionellen Wahrnehmung des Alevitentums leiten ließ und eine staatsnahe Haltung in Bezug auf die Türkei einnahm. Während sich in den Räumlichkeiten der HBKC auf Türkei-Flaggen und Bilder von Atatürk stoßen lässt, sind diese Objekte im Berliner AAKM nicht zu sehen, da man hier der Meinung ist, dass dies zu einer Spaltung zwischen Kurden und Türken führen würde (Massicard, 2007: 304). ${ }^{48}$ Kehl-Bodrogi merkt an, dass die Entscheidung des Berliner Bildungsministeriums vom April 2002, alevitischen Religionsunterricht an Schulen stattfinden zu lassen, in den Zeitungen und in der Öffentlichkeit als "Liberaler Koranunterricht an Schulen" wahrgenommen wurde, da die Aleviten und die Berliner AAKM, die die Belange der Aleviten in Berlin vertritt, mit der "Linken" in Verbindung gebracht und als Alternative zu dem seitens des Landes Berlin als repräsentatives Organ sunnitischer Muslime anerkannten Islamrat (Islamischen Föderation Berlin e.V.) gesehen wurden (Kehl-Bodrogi, 2003: 464467; 2002: 64). All dies hat dafür sorgt, dass Berlin einen besonderen Platz in der alevitischen Institutionalisierung in Deutschland hat. Tatsächlich haben die Berliner Aleviten, wie aus den Äußerungen von Kehl-Badrogi hervorgeht, insbesondere

47 Für detaillierte Informationen siehe Kosnick, 2008: 120-123.

48 Auch Kehl-Bodrogi kommt zu ähnlichen Erkenntnissen. Siehe Kehl-Bodrogi, 2002: 54-57. 
durch den alevitischen Religionsunterricht wesentlich dazu beigetragen, dass das Alevitentum in der deutschen Öffentlichkeit wahrgenommen wird und auch der Unterschied zwischen ihnen und dem sunnitischen Islam deutlich wird.

\subsection{Köln}

Während im Rahmen dieses Artikels gewiss nicht die Entstehungsprozesse sämtlicher kulturzentrierter Einrichtungen abgehandelt werden können, kann als ein letztes Beispiel für solche Einrichtungen das Alevitische Kulturzentrum in Köln angeführt werden. Da eine Gruppe von Aleviten, die sich unter anderem aus Mahmut Gülçiçek, Ali Rıza Gülçiçek, Gülizar Cengiz, Hasan Cengiz, Necdet Saraç und Remzi Sofuoğlu zusammensetzte, den Verein zur Förderung der Hacı Bektaş Veli Kultur in Köln und Umgebung für unzureichend im Hinblick auf die Institutionalisierung des Alevitentums hielt, wollte sie unabhängig weiterarbeiten. Zu diesem Zweck trafen sie Vorbereitungen, in Köln eine Veranstaltung ähnlich der von der Alevitischen Kulturgruppe Hamburg ausgerichteten Kulturwoche zu organisieren. Ali Riza Gülçiçek gibt als Grund für diese Mobilisierung in Köln die Begegnung mit dem Journalisten Necdet Saraç an und sagt, dass ihn diese Bekanntschaft in die alevitische Bewegung gezogen habe. Anders als in Hamburg zog die Kölner Gruppe es vor, statt von einer Woche lieber von Tagen zu sprechen, und beschloss, unter dem Namen Anatolisch-Alevitische Kulturtage für den 23., 24. und 28. November 1991 ein Veranstaltungsprogramm aufzusetzen. In Rahmen des dreitägigen Programms traten zahlreiche Künstler wie z.B. Arif Să̆, Ozan Emekçi, Hasret Gültekin, İhsan Güvercin und Yusuf Güvercin auf. Mit dem Theaterstück "Ich sah aus wie Yunus" trug auch das Kölner Freundestheater zu den Anatolisch-Alevitischen Kulturtagen bei, an denen außerdem diverse Podiumsdiskussionen und Konferenzen stattfanden, bei denen Fuat Bozkurt, İlhan Selçuk, Nejat Birdoğan und vor allem Irene Melikof die Besucher über das Alevitentum informierten. Wie in Hamburg und Berlin kam auch in Köln nach den Alevitischen Kulturtagen die Idee eines Vereins auf, die am am 23. Februar 1992 mit der Gründung des Kölner AKM verwirklicht wurde (Gülçiçek, 2018: 55,56,60). ${ }^{49}$ Die Alevitischen Kulturtage wurden auch nach der Gründung des Kölner AKM noch einige Jahre lang fortgesetzt.

49 Ohne Einzelheiten zu nennen, berichtet Zeynel Gül, dass die in Hamburg begonnene Tradition der alevitischen Kulturwoche in Berlin, Köln und Rheda-Wiedenbrück fortgesetzt wird und dass etwa 20.000 Menschen an diesen Veranstaltungen teilnehmen. Siehe Gül, 1999: 94, 95. 
Dettling beobachtet, dass viele gläubige Aleviten begannen, sich von den alevitischen Institutionen abzuwenden, weil sie sich an den politischen Debatten in den Vereinen störten und nicht an politischen Konflikten beteiligt sein wollten (2006: 34). Auch Tosuns Erzählungen stützen diese Beobachtung. So schlug Hasan Kllavuz beispielsweise während der Ausarbeitung der Satzung im Rahmen des Gründungsverfahrens der Hamburger AKM vor, die Satzung auch in kurdischer Sprache zu verfassen, und wurde darin von Ali Haydar Cilasun unterstützt, der später den Posten des Vorsitzenden der Alevitischen Union Kurdistans bekleiden sollte. Als sein Vorschlag abgelehnt wurde, verließ Kılavuz die Gründungsversammlung. Später trat er dem Verein jedoch als Mitglied bei und hatte nach 1996 drei Amtszeiten lang den Vorsitz inne (Tosun, 2002: 113-117; Özbaş 1999: 45,46). ${ }^{50}$ Aleviten, deren Priorität der Glaube ist, stören sich an nationalistischen und politisch-separatistischen Strömungen in den Vereinen.

\section{Fazit}

Die in Deutschland entstandenen Organisationen der Aleviten weisen eine zweigliedrige Struktur auf. Während der erste Typ von Organisationen in diesem Artikel als "Dede-zentriert" bezeichnet wird, weil diese um die Dedes, die im Alevitentum als Glaubensführer gelten, herum entstanden sind, wird der zweite, überwiegend von Aleviten aus dem linken Spektrum getragene Typ als "kulturzentriert" bezeichnet. Die insbesondere von Martin Sökefeld vertretene und andernorts vielfach zitierte Behauptung, die alevitische Bewegung sei von linken Aleviten in Hamburg ausgegangen, ist nicht haltbar. Dies liegt zum einen daran, dass der erste alevitische Verein in Hamburg erst 1990 gegründet wurde, als zu anderen auch daran, dass die Mehrheit der linken Aleviten - mit Ausnahme der wenigen, die sich innerhalb kulturzentrierter Organisationen zusammenschlossen eine alevitische Institutionalisierung zunächst ablehnte, weil sie befürchteten, dass sich dies zum Nachteil linker Organisationen auswirken würde. Tatsächlich fand, wie in diesem Artikel ausführlich dargelegt wurde, die erste offizielle Gründung eines alevitischen Vereins 1987 in Köln statt. Hierbei handelte es sich um eine Institution, in deren Zentrum der Glaube stand und die von den Dedes ins Leben gerufen wurde,

50 Hasan Kllavuz soll Mitglied der Deutschen Föderation der Arbeitervereinigungen Kurdistans, kurz KOMKAR, gewesen sein, die ihre Aktivitäten in Deutschland 1979 in Verbindung mit der Sozialistischen Partei Kurdistans aufnahm, die im Dezember 1974 unter der Führung von Kemal Burkay gegründet wurde. Zur KOMKAR siehe Franz, Oktober 202M.S.: 22. 
die darauf bedacht waren, Probleme mit dem türkischen Staat zu meiden und sich von ideologischen Auseinandersetzungen fernzuhalten. Köln war zudem der Ort, wo in der Folge das Kölner AKM als eine der ersten kulturzentrierten Organisationen gegründet wurde. Will man das Zentrum der alevitischen Bewegung also sowohl geografisch als auch namentlich verorten, sollte die Wahl in Anbetracht der Tatsache, dass die Stadt Schauplatz beider Seiten des alevitischen Institutionalisierungsprozesses war und nach wie vor Sitz der AABF ist, eher auf Köln und die um Dedes wie Şinasi Koç, Niyazi Bozdoğan und Hüseyin Tur herum entstandenen Strukturen fallen. Dementgegen scheint die Dominanz der Hamburger Organisationen dazu geführt zu haben, dass die ersten Institutionalisierungserfahrungen, die um die Dedes herum organisiert waren, oft übersehen werden. 
AKGÜN, Sibel (2012) "Kimlik Oluşumu Sürecinde Örgüt ve Örgütlenmenin Yeri”. Turkish Studies. 2018/13/3. 1-12.

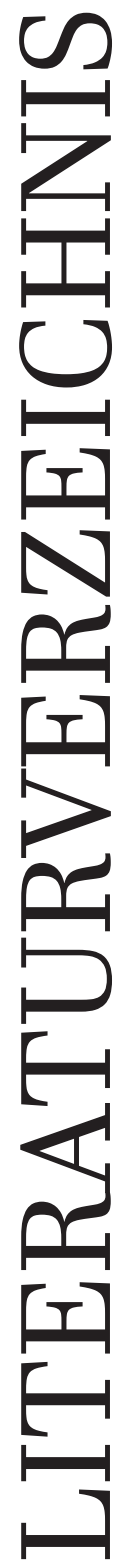

AKSOY, Erdal - TEKTEN AKSÜRMELI, Zeynep Serap (2018) "Diaspora İçinde Diaspora: Almanya Alevileri”. Türk Kültürü ve Hacı Bektaș Veli Araștırma Dergisi. 2018/85.

AKSÜNGER, Handan (2013). Jenseits Des Schweigegebots Alevitische Migrantenselbstorganisationen Und Zivilgeselschaftliche Integration In Deutschland Und Den Niederlanden. Band 11 Cilt. Münster/New York/München/Berlin: Waxmann Verlag Gmbh.

AKSÜNGER, Handan (2013a) "Gemeinschaftsbildung Und Integration. Die Aleviten In Deutschland Und Den Niederlanden". Migrantenorganisationen, Engagement, Transnationalität Und Integration. Hrg. Günther Schultze - Dietrich Thränhardt. Bonn: Friedrich Ebert Stiftung. 42-54.

AKYÜZ, Niyazi (2007). Dinin Örgütsel İklimi: Dini Gruplar. Ankara: Gündüz Eğitim ve Yayıncilık.

ARSLAN, Zeynel (1997). "Kilisede Dede Nikahı". Alevilerin Sesi. 1997/21. 37 39.

ATAMAN, Nurgül et al. (Hrsg.) (2019). Yol Bir Sürek Binbir, Einheit in Vielfalt. Köln: Alevitische Gemeinde Deutschland e.V.

AYDOĞDU, Uğur ve AYDEMIR, Ahmet (2013). Avrupa Alevi Yapılanmast Kronoloji 1989-1998. Ankara: Öztepe Matbaacilık Ltd.Şti.

BAHADIR, İbrahim (2002). Cumburiyet'in Kurulus Sürecinde Atatürk ve Aleviler. Ankara: Kalan Yayınları.

BOZKURT, Tufan (2015). Mannheim ve Cevresinde Yasayan 3. Kusak Alevi gençlerinin Alevilike, Türkiye Ve Almanya Algzlayışlan Alan Araşstrması. Istanbul: Marmara Universität, Institut für Nah- und Mitteloststudien, Soziologie und Anthropologie des Nahen und Mittleren Osten, Master-Thesis.

BÜYÜKGÖL, Halit (2005). "Berlin AAKM 25 yaşında”. Alevilerin Sesi. 2005/84. 43.

BÜYÜKGÖL, Halit (2005). "Yurtseverler Birliği'nden AAKM-Cemevi'ne uzanan yol". Alevilerin Sesi. 2005/81. 42.

CABA, Mehmet (2014). Avrupa Alevi Örgütlenmesi. Ankara: İmla Kitap Yayınları. CEVAHIR, Muhsin (2008). Geçmişten Geleceğe. Çorum: Kardeș Matbaacılık.

CEYLAN, Yılmaz (2015). "Yeni Bir Kimlik Hareketi: Anadolu Aleviliği”. Dicle Üniversitesi Sosyal Bilimler Enstitüsü Dergisi. 2015/13. 210-226.

DAĞYELİ, Jeanine Elif (2006). "Die Aleviten - eine Glaubensgemeinschaft aus der Türkei". Islamisches Gemeindeleben in Berlin. Ed. Alexa Färber - Riem Spielhaus. Berlin: Beauftragte des Senats von Berlin für Integration und Migration. 75-78

DETTLING, Wilfried (2006). Das Religionsverständnis der anatolischen Aleviten in Deutschland. Roma: Pontificia Università Gregorianafacoltà Di Missiologia Dissertazione per il Dottorato, unveröffentlichte Doktorarbeit.

DİKMEN, Seyit Ali (2005). "İlk Alevilik Öğretmenleri Mezun Oluyor". Alevilerin Sesi. 2005/81. 43. 
ENGIN, Havva (2015). "Das Alevitentum - der vorderasiatische 'Kultur-Islam"'. Christentum und Islam in Deutschland: Grundlagen, Erfahrungen und Perspektiven des Zusammenlebens. Hrsg. Mathias Rohe et al. 96-137. Schriftenreihe / Bundeszentrale für politische Bildung 1634. Bonn: bpb, Bundeszentrale für politische Bildung, Sonderausgabe.

ENGİN, İsmail (2001). "Diaspora'da Aleviler ve Aleviliğin Geleceği: Nereden Nereye". Pir Sultan Abdal. 2001/43. 83-87.

ERTAN, Mehmet (2017). Aleviliğin Politikleşme Süreci Kimlik Siyasetinin Kısıthllklar ve Imkanlar. Istanbul: İletișim Yayınları.

FRANZ, Erhard (2002). Exilopposition als politischer Akteur (I): Oppositionsgruppen aus der Türkei. Deutsches Orient-Institut im Verbund Deutsches Übersee-Institut.

GREIL, Arthur L. und DAVIDMAN, Lynn (2016). "Kimlik ve Din”. übers. v. Abdullah Özbolat Saffet Kartopu. Kimlike ve Din. Hrsg. Abdullah Özbolat - Mustafa Macit. Adana: Karahan Kitabevi. 3-38.

GÜL, Zeynel (2002). "Almanya'da Alevi Örgütlenmesi Üzerine Kısa Bir Bakış”. folklor/edebiyat Alevilik Örel Sayıst - 2002/2/30. 371-382.

GÜL, Zeynel (1999) Dernekten Partiye Avrupa Alevi Örgütlenmesi. Ankara: İtalik Kitapları.

GÜL, Zeynel (1995). Yolmuyuг, yolcu mu? Alevi Örgütlenmesi nasıl basslad, nasil gelisti ve nereye gidiyor. Essen: Emek Druckerei.

GÜLÇIÇEK, Ali Rıza (2018). Simırlar Așan Mücadelem, Karacaören-Köln-Ankara. Ankara: İtalik Kitaplar1.

GÜMÜŞ, Burak (2007). Die Wiederkehr des Alevitentums in der Türkei und in Deutschland. Konstanz: Hartung-Gorre Verlag.

GÜNAY, Ünver (2012). Din Sosyolojisi. Istanbul: İnsan Yayınları.

IRFAN, Erdal (1994). "İlk Alevi Televizyonu: AL-Canlar TV”. Alevilerin Sesi. 1994/5. 17.

KAÇMAZ, Metin (2018). Almanya Alevi Örgütlenmesi Taribi 1989-2018. Köln: YOL Bilim Kültür Araştırma Yayınları.

KAPLAN, Doğan (2014). "Aleviliğin Dayandığı Etnik Kimlik/ler Üzerine”. Geçmisten Günümüze Alevilik I. Uluslararası Sempozyumu, 03-05 Ekim 2013 Bingöl: Bingöl Üniversitesi Yayınları. 441-447. KAPLAN, İsmail (2009). Alevice, Inancme ₹ve Direncimi\%. Köln: Almanya Alevi Birlikleri Federasyonu Yayınlar1.

KAPLAN, Ismail (2004). Das Alevitentum: Eine Glaubens- und Lebensgemeinschaft in Deutschland. Köln: Alevitische Gemeinde Deutschland e.V.

KAPPHAN, Andreas (2006). "Zugewanderte aus islamisch geprägten Ländern in Berlin". Islamisches Gemeindeleben in Berlin. Hrsg. Alexa Färber - Riem Spielhaus. Berlin: Beauftragte des Senats von Berlin für Integration und Migration. 18-23.

KEHL-BODROGI, Krisztina (1996). "Tarih mitosu ve kollektif kimlik". Birikim Ayllk Sosyalist Kültür Dergisi. 1996/88. 52-63.

KEHL-BODROGI, Krisztina (2003) "Von Der Geheimen Glaubenslehre zur Öffentlichen Religion Alevitischer Religionsunterricht in Deutschland". Bilgi Toplumunda Alevilik. Hrsg. İbrahim Bahadır. Ankara: Bielefeld Alevi Kültür Merkezi Yayınları. 453-473.

KEHL-BODROGI, Krisztina (2006). "Von der Kultur zur Religion Alevitische Identitätspolitik in Deutschland". Max Planck Institute For Social Anthropology Working Papers. 2006/84. 1-20.

KEHL-BODROGI, Krisztina (2002). "Was du auch suchst, such es in dir selbst!" Aleviten (nicht nur) in Berlin. Berlin: Die Ausländerbeauftragte des Senats. 
KELEŞ, Bülent (2012). Almanya'da Islam Ve Müslümanlar “Bavyera Örneği”. Sivas: Cumhuriyet Universität, Institut für Sozialwissenschaften, Islamwissenschaften, Islamische Glaubensgeschichte, unveröffentlichte Master-Thesis.

KELEŞ, Bülent (2020). "Kurumsal Kimlik Kazanması Bağlamında Yurtdışı Alevi Örgütlenmesi 'Almanya Örneği”". Uluslararası Hacı Bektaş Veli ve Takipçileri Sempozyumu Bildiriler Kitabı Hrsg. A. Yılmaz Soyyer. Istanbul: Türk Ocağ1 Yayınları. 391-423.

KORKMAZ, Bülent (2017). Die Bewohner von Rız̨a Şehri. Duisburg: Alevitische Gemeinde Duisburg e.V.

KOSNICK, Kira (2008). "Mit eigener Stimme? Migrantische Medien und alevitische Strategien der Repräsentation". Aleviten in Deutschland, İdentitätsprozesse einer Religionsgemeinschaft in der Diaspora. Hrsg. Martin Sökefeld. Bielefeld: transcript Verlag. 109-132.

KULA, Onur Bilge (2012). Almanya'da Türk Kültürü Cok-Kültürlülük, ve Kültürlerarası Eğitim. übers. v. Aytekin Keskin. İstanbul: İstanbul Bilgi Üniversitesi Yayınları.

KÜÇÜK, Hülya (2003). Kurtuluș Savaşı’nda Bektașiler. Istanbul: Kitap Yayınevi.

LANGER, Robert (2006). "Das Reframing Alevitischer Gemeinderituale im Kontext von Ritualtransfer und Migration”. Rituale im Bewegung, Rahmungs- und Reflexivitätsprozesse in Kulturen der Gegenwart. Hrsg. Henrik Jungaberle - Jan Weinhold. Berlin: LIT Verlag. 129-144.

LANGER, Robert (2010). “Hacı Bektaş Veli’nin Almanya’ya Varışı: Göç ve Diaspora Çerçevesinde Yurtdışındaki Alevilikte Dinsel Ritüel Dönüşüm-Değişimler”. Nevşehir: Atatürk Kültür Merkezi. 379-388

LANGER, Robert (2015). “Marjinalleştirilen İslam’: Kursal Ritüellerin Kentsel ve Çoğulcu Bağlamlara Aktarımı ve Uluslar Ötesi 'Uygulama Toplulukların' Ortaya Çıkışı”. über. v. İrem Wedekind. Kı̨̧lbaşlık, Alevilik, Bektaşilik Tarih-Kimlik-Innanç Ritüel. Hrsg. Yalçın Çakmak - İmran Gürtaş. Istanbul: İletişim Yayınları. 481-502.

MADEN, Fahri (2015). "En Uzun Yüzyılda Bektaşilik ve Bektaşiler”. Kızılbaşlık, Alevilik, Bektaşilik: Tarih, Kimlik, Inanç, Ritüel. Hrsg. Yalçın Çakmak - İmran Gürtaş. Istanbul: İletişim Yayınları. 185213.

MANDEL, Ruth (2000). "Yabanc1 Ortamlarda Alevi-Bektaşi Kimliği Berlin Örneği”. Türk Kültürü ve Hacı Bektaş Velî Araştırma Dergisi. 2000/14. 59-67.

MASSİCARD, Élise (2007). Türkiye'den Avrupa'ya Alevi Hareketinin Siyasallasması. übers. v. Ali Berktay. Istanbul: İletişim Yayınları.

MÉLIKOFF, Irène (2006). Uyur İdik Uyardılar: Alevilik-Bektaşilik. Araştırmalar. übers. v. Turan Alptekin. İstanbul: Demos Yayınları.

OKAN, Murat (2004). Türkiye'de Alevilik: Antropolojik Bir Yaklaşım. Ankara: İmge Kitabevi.

ÖZ, Baki (1990) Kurtulus Savaşı'nda Alevi-Bektaşiler. Istanbul: Can Yayınları.

ÖZBAŞ, M. Deniz (Hrsg.) (1999). Onlarn Öyküsü, Hamburg Alevi Kültür Merkezi Taribi. Hamburg: Hamburg Alevi Kültür Merkezi-Alevitisches Kulturzentrum Hamburg e.V.

PERŞEMBE, Erkan (2005). Almanya'da Türk Kimliği Din ve Entegrasyon. Ankara: Araştırma Yayınları. RITTERSBERGER-TILIÇ, Helga (2010). “Almanc1' Kimliğinin Alevi Kimliğine Dönüştürülmesi”. Çev. Bilge Kurt Torun - Hayati Torun. Alevi Kimliği. Hrsg. Tord Olsson et al. Istanbul: Tarih Vakfı Yurt Yayınlar1. 91-103.

SALMAN, Cemal (2015). Gös ve Kentleşme Sürecinde Alevi Kimliğinin Kültürel-Siyasal Değisimi ve Dönüsümü. Istanbul: Istanbul Universität, Institut für Politikwissenschaften, Politikwissenschaften und Öffentliche Verwaltung, Doktorarbeit. 
SALMAN, Cemal (2017). "Türkiye Göç ve Kentleşme Süreçlerinde Alevi Deneyiminin Yeri: Bir Dönemlendirme Önerisi”. Gazi Üniversitesi İktisadi ve İdari Bilimler Fakültesi Dergisi Gazi Akademi Genç Sosyal Bilimciler Sempozyumu 2017 Sonderausgabe. 24-51.

SOYYER, A. Yılmaz (2005). 19. Yürynlda Bektaşı̂lik. Izmir: Akademi Kitabevi.

SÖKEFELD, Martin (2002). "Alevi Dedes In The German Diaspora: The Transformation Of A Religious Institution". Zeitschrift Für Ethnologie. 2002/127. 163-186.

SÖKEFELD, Martin (2003). "Alevis In Germany The Politics Of Recognition”. New Perspektives On Turkey. 2003/28-29 133-161.

SÖKEFELD, Martin (2008c). “Almanya'da Aleviler 'Almanya'da Müslümanlar' Anketinden Edinilen Verilere Dair Yorum”. Din Monitörü 2008 Almanya'daki Müslümanlarda Dindarlke Dini Düşünce Ve Pratiklere Bir Bakııs. Osnabrück: Bertelsmann Stiftung. 32-37.

SÖKEFELD, Martin (2015). "Almanya'da Aleviler; Takiyeden Alevi Hareketine”. Kızılbaşlık, Alevilik, Bektaşilik Tarib-Kimlik-Inanç Ritüel. Hrsg. Yalçın Çakmak - İmran Gürtaş. Istanbul: İletişim Yayınlar1. 397-422.

SÖKEFELD, Martin (2008b). "Einleitung: Aleviten In Deutschland - Von Takiye Zur Alevitischen Bewegung”. Aleviten In Deutschland, İdentitätsprozesse Einer Religionsgemeinschaft In Der Diaspora. Hrsg. Martin Sökefeld. Bielefeld: Transcript Verlag. 7-36.

SÖKEFELD, Martin (2008a). "Sind Aleviten Muslime? Die Alevitische Debatte Über Das Verhältnis Von Alevitentum Und Islam In Deutschland". Aleviten In Deutschland, İdentitätsprozesse Einer Religionsgemeinschaft In Der Diaspora. Hrsg. Martin Sökefeld. Bielefeld: Transcript Verlag. 195218.

SÖKEFELD, Martin (2003a). 'Wir Sind Auch Da!' Aleviten In Hamburg Und Der Kampf Um Anerkennung". Wir Sind Auch Da! Über Das Leben Von Und Mit Migranten In Europäischen Grossstädten. Hrsg. Angelika Eder. Forum Zeitgeschichte. Hamburg: Dölling Und Galitz. 14/243-265.

SPIELHAUS, Riem (2006). "Organisationsstrukturen islamischer Gemeinden". Islamisches Gemeindeleben in Berlin. Ed. Alexa Färber - Riem Spielhaus. Berlin: Beauftragte des Senats von Berlin für Integration und Migration. 12-17.

ŞAHHÜSEYINOĞLU, H. Nedim (2001). Alevi Örgütlerinin Taribsel Süreci. Ankara: İtalik Kitapları/ Ayyıldız Yayınları.

ŞAHIN, Şehriban (2002). "Bir Kamusal Din Olarak Türkiye'de ve Ulus Ötesi Sosyal Alanlarda İnşa Edilen Alevilik". folklor/edebiyat. 2002/VIII/XXIX. 123-162.

ŞENER, Cemal (1994). Atatürk, ve Aleviler. Istanbul: ANT Yayınları.

ŞENER, Cemal - İLKNUR, Miyase (1995) Krrklar Meclisinden Günümüze Alevi Örgütlenmeleri: Şeriat ve Alevilik. Istanbul: ANT Yayınlar1.

TOSUN, Halis (2002). Alevi Kimliğiyle Yasamak. Istanbul: Can Yayınları.

TÖNNIES, Ferdinand (1887). Gemeinschaft und Gesellschaft. Leipzig: Fues's Verlag (R. Reisland).

TUR, Seyit Derviş (2016). Avrupa'da Illk. Alevi Yapılanmasmm Taribi Gergekeleri ve Seyit Derviș Tur'un Hatiralar. İtimat Ofset Matbaa.

TÜRKDOĞAN, Orhan (2007). Türk Toplumunun Kültürel Dinamikleri. Istanbul: Kum Saati Yayınları. ÜÇER, Cenksu (2010). "Geleneksel Alevilikte İbadet Hayatı, Bazı Adab ve Erkan”. Anadolu'da Alevilï̆in Dünü ve Bugïnü. Hrsg. Halil İbrahim Bulut. Sakarya: Sakarya Üniversitesi. 457-510.

YAPICI, Asım (2016). "Dini Grup Kimliği: Kimlik ve Dini Aidiyet”. Kimlik ve Din. Hrsg. Abdullah Özbolat - Mustafa Macit. Adana: Karahan Kitabevi. 41-64. 
YAŞAR, Mehmet Ali - KORKMAZ, Bülent (Hrsg.) (2009). Alevitische Gemeinde Duisburg e.V, Duisburg Alevi Toplumu: Alevitentum, Alevilik. Duisburg: Alevitische Gemeinde Duisburg e.V.

YILDIRIM, Rıza (2012). "Geleneksel Alevilikten Modern Aleviliğe: Tarihsel Bir Dönüşümün Ana Eksenleri”. Türk Kültürü ve Hacı Bektas Velî Araștırma Dergisi. 2012/62. 135-162.

YÜREKLIK, Güner (1991). “Berlin'de Alevi haftası”. Cumburiyet Gą̧etesi. (31. März 1991).

ZIRH, Besim Can. "Bir Kesişim Kümesi Olarak Milli-Muhafazakarlığın üç Değili: Aleviler, Ermeniler, Kürtler”. Kı̨̧lbaşhlk, Alevilik, Bektaşilik: Tarih, Kimlik - Inanç-Ritüel. Hrsg. Yalçın Çakmak - İmran Gürtaş. Istanbul: İletişim Yayınları. 327-368.

“AABF Genel Başkanı Turgut Öker: 'Alevilik Bir Dünya İnanc1"'. Alevilerin Sesi. 40 (November 2000). 15-21.

“Alevilik Bildirgesi”. Hamburg Alevi Kültür Grubu, Mai 1989. Hamburg Alevi Kültür Grubu.

“Alevilik Tarihe Karışıyor: Dinsel, Kültürel ve politik bir yok oluşun öyküsü...” Nokta 5/38 (27. September 1987).

“Duisburg Alevi Kültür Merkezi 10. Y1lını Kutladı”. Alevilerin Sesi. 29 (Oktober 1998), 48.

“Gerçek İlim’i Yayınlayan Birlikler”. Gerçek İlim. 61/62/63 (September 1994), 39.

"Müjde Berlin Anadolu Alevileri Kültür Merkezi Cemevi'ne Kavuştu”. Alevilerin Sesi. 29 (Oktober 1998), 50 .

Satzung der Mainz Bektaschi-Gemeinde e.V. Satzung. 5.

Ablener Volkszeitung. "Türkische Aleviten mit Fest in der F.-Ebert-Halle” (18. Januar 1988).

\section{Interview}

Derviş Tur (1936). Rüsselsheim, Mittelschulabschluss, Rentner, Dede, "Interview vom 13.12.2017” Niyazi Bozdoğan (1939). Köln, Mittelschulabschluss, Rentner, Dede, "Interview vom 08.06.2020”. Sabit Yildız (1944). Rodgau, Mittelschulabschuluss, Rentner, Talip, “Interview vom 23.01.2019”. 